\title{
Hierarchical mathematical models of complex plants on the basis of power boiler example
}

\author{
WŁODZIMIERZ STANISŁAWSKI and MAREK RYDEL
}

\begin{abstract}
The methodology of hierarchical linearized mathematical models construction of complex plants to control purposes is presented in the paper. Thanks to the methodology, the one high order model (flat, one level model), is replaced by a collection of models, which are placed at different hierarchy levels. The models represent dynamic processes typical for each hierarchy level, and omit fast dynamic processes significant at lower levels. The higher is hierarchy level, the slower dynamic processes is described by the model and the lower is order of the models. Multi-level model structure gives possibility of dynamic properties analysis by application of aggregation procedure. One of the principal aggregation procedure is reduction of models at individual levels of hierarchical structure. Such approach enables creating a reduced hierarchical model including a collection of models at every level of hierarchy, characterized by various adequacy scopes and accuracy of the plant features approximation. The paper presents methodology of hierarchical complex plants models creation on the example of evaporator of the BP1150 boiler. Each of the subsystem at individual level of model hierarchy is a multi-input and multi-output causal systems.
\end{abstract} tion

Key words: once-through steam boiler, reduced hierarchical model, complex model reduc-

\section{Introduction}

Very intensive increase of calculation capacity of computers is observed in last years as well as a development of software for complex plants modeling and simulation, which results in a fast growth of mathematical models complexity. Particularly significant for the models complexity is development of programmes for modeling of distributed parameter systems by means of the finite elements method. Such programmes automatically generate thousands of finite elements, and obtained mathematical models include thousands of state variables. This high complexity of mathematical model involves long computer simulation times, significant demand for processors' calculation capacity and numerical problems which appear especially with highly stiffness models.

To simplify building models of the complex plants it is necessary to apply a multi level (hierarchical) structure of the model and its decomposition into a series of subsys-

The Authors are with Institute of Control and Computer Engineering, Opole University of Technology, Sosnkowskiego 31, 45-272 Opole, Poland. E-mails: w.stanislawski@ po.opole.pl, m.rydel@ po.opole.pl

Received 09.07.2010. Revised 15.11.2010. 
tems which are simpler to be modeled. The key issue of such model construction is the fact, that only the lowest (zero) level subsystems are described by a set of differential and algebraic equations. At all higher levels the model includes subsystems of directly lower level with topology of connections between them.

Simulation programmes (e.g. MATLAB/Simulink) make the technology of hierarchical models construction possible, by simple linking of causal subsystems. It should be noted however, that hierarchical construction of models in calculation environment is related solely with the functionality of a graphic editor (nesting and masking operations of subsystems models). In the computer memory, the model is stored as a system of algebraic and ordinary differential equations. Such model is very complex and difficult to be analyzed due to its high order, therefore it is impossible to draw a conclusion about dynamic properties of such system.

Multilevel model structure with models decomposition on individual level of hierarchical structure gives a possibility of the plant dynamic properties analysis by means of aggregation procedure. Aggregation is opposed to the decomposition. One of the principal aggregation procedure is reduction of models at individual level of hierarchical structure. This approach enables creating a reduced hierarchical model which includes a collection of models at every level of hierarchy, characterized by various adequacy scopes and accuracy of the plant properties approximation [25].

During reduction, the primary model is approximated by a lower order model, which assures the required approximation accuracy. The reduction operation allows for eliminating complex phenomena and dynamic processes which are not significant at a given hierarchical level. Therefore reduced model omits part of phenomena which appears in the actual plant. It is characterized with a limited adequacy scope. In linear case it is determined by a range of frequencies in which the model approximates the plant's properties with determined accuracy. For instance zero frequency is the lowest one of the adequacy scope for the control plants models, which points the accordance of the reduced models with primary models in steady state.

Reduced mathematical models, adequate in a limited frequency range are also necessary due to:

- shortening of the computer simulation time,

- facilitating application of the models in a real time control systems,

- application for design of control systems.

\section{Concept of hierarchical model construction}

Plant model decomposition into a series of simple subsystems is widely applied method of complex plants and control systems modeling. The decomposition procedure is carried out as long as determining of the lowest (zero) level subsystems models is 
possible and feasible. Graph in Fig. 1 illustrates the complex plant model decomposition diagram ( $L$ refers to absolute number of model levels, whereas $l$ refers to relative one).

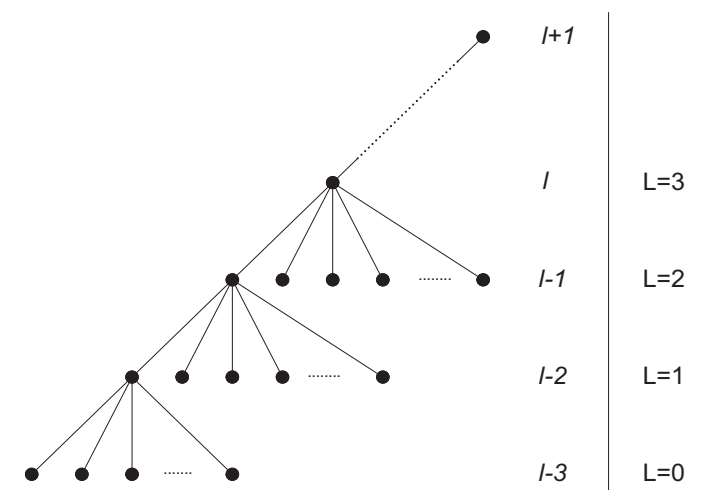

Figure 1. Complex plant subsystems model structure

As a rule, the procedure of decomposition is not complex. Simulation programmes (e.g. MATLAB/Simulink) make the technology of hierarchical models construction possible, by simple linking of causal subsystems. Only the zero level subsystems are described by a set of differential and algebraic equations. At all higher levels the model includes subsystems from the lower level with a given structure of connections. On this basis the model on $l$-th level may be presented as a set of subsystems models of level $l-1$, with the following topology of connections between the subsystems:

$$
S_{l}=\left\langle S_{l-1}^{i}, P_{l}\right\rangle ; \text { for } i=1,2, \ldots, n_{l-1}
$$

where: $n_{l-1}$ - number of subsystems on $l-1$-th level of hierarchy, $P_{l}$ - set of connections between subsystems, at level $l$.

Individual subsystems at level $l-1$ are causal systems, with input vector $U_{l-1}^{i}=\left[u_{l-1}^{i, 1}, u_{l-1}^{i, 2}, \ldots, u_{l-1}^{i, m}\right]$, output vector $Y_{l-1}^{i}=\left[y_{l-1}^{i, 1}, y_{l-1}^{i, 2}, \ldots, y_{l-1}^{i, p}\right]$ and state vector $X_{l-1}^{i}=\left[x_{l-1}^{i, 1}, x_{l-1}^{i, 2}, \ldots, x_{l-1}^{i, n}\right]$. Every subsystem is described by a transition and output operators of $H_{l-1}^{i}, G_{l-1}^{i}$ :

$$
\begin{aligned}
& X_{l-1}^{i}=H_{l-1}^{i}\left(U_{l-1}^{i}\right) \\
& Y_{l-1}^{i}=G_{l-1}^{i}\left(U_{l-1}^{i}, X_{l-1}^{i}\right) .
\end{aligned}
$$

Connections of individual subsystems at level $l$ may be described by means of various methods, especially with:

- block diagram (graphic method of model structure representation at level $l$ ),

- zero-one connections matrix. 
Model decomposition at a given hierarchy level can be carried out in any way, considering a series of criteria:

- technological structure of the plant (division into individual technological devices),

- status of a working media (e.g. steam, water, two-phase medium etc.),

- physical, chemical and other phenomena within the plant.

It may be stated in general, that the model creator determines individual subsystems and defines the assumed borders between them.

The basic feature of a hierarchical model is the fact, that the model includes a whole series of models at individual levels of the hierarchy. At every level we meet aggregated models obtained on the basis of subsystem models of the lower level. As a result of aggregation, the model of a given level includes individual subsystems models properties, and takes into account the connections between the subsystems, especially the feedbacks. Moreover, the aggregated model at a given level of hierarchy includes only the dynamic processes which are significant at this level, and omits the dynamic processes which are significant at the lower hierarchy levels (these are usually faster processes). For instance the mathematical model of a steam boiler does not take into account sound phenomena (pressure changes displacement) in the evaporating tubes.

The subsystems models have got a precisely determined adequacy scope at individual hierarchy levels, which for the linearized models is defined by the limit frequency

$\omega_{\text {max }}$. The scope of adequacy of the subsystem model at a given hierarchy level results from the scopes of lower level subsystems adequacy and requirements (e.g. related to the control systems design).

\section{Models aggregation}

Aggregation may be understood as a conclusion about the model properties at a given hierarchy level, on the basis of subsystems models of the level directly below it and the topology of connections between them [25, 28]. Models of higher hierarchy levels subsystems are aggregated and represent significant properties for a given level. Model aggregation can be carried out in two ways, by application of the reduction procedure or by model abstraction at individual hierarchy levels. Using the first and the second procedure at a given hierarchy level, the model of simplified structure is obtained if compared with the model obtained by connection of lower level subsystem models [31].

As a result of reduction, in the reduced model, the set of input variables remains unchanged if compared with the primary model. However, the state coordinates change, so that the reduced model outputs reflect the outputs of the primary model with assumed accuracy (Figure 2) [1]. 
As a result of abstraction, the lower order model is constructed, which is characterized by other inputs, outputs and state vector [22]. The purpose of abstraction is to determine transformation of the state vector $x$ into vector $g=P x$, so that the new model (called abstraction) has got given properties if and only if the primary model is characterized by determined properties. It is referred to propagation from macro model (abstraction) to micro model (primary model) [22] (Fig. 2).

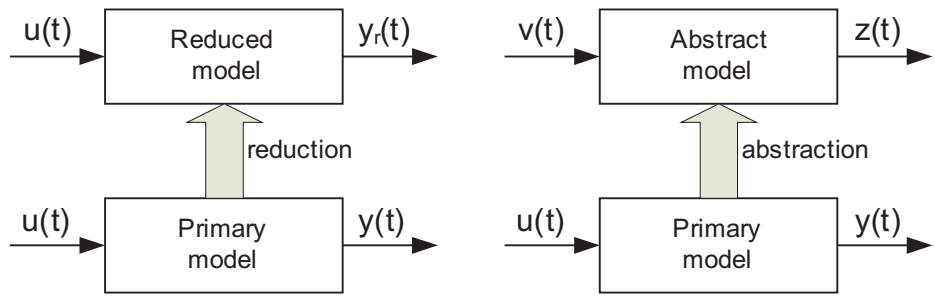

Figure 2. Idea of model reduction and abstraction

The task of linear model reduction can be presented as follows: according to the stable (or unstable) model of order $n$ (3), a reduced model of order $k$ should be determined (4), where $k<n$, such that the determined approximation error norm $\left\|y(t)-y_{r}(t)\right\|$ reaches the minimum value. The respective models can be expressed as follows:

$$
\begin{aligned}
\dot{x}(t) & =\mathbf{A} x(t)+\mathbf{B} u(t) \\
y(t) & =\mathbf{C} x(t)+\mathbf{D} u(t) \\
\dot{x}_{r}(t) & =\mathbf{A}_{\mathbf{r}} x_{r}(t)+\mathbf{B}_{\mathbf{r}} u(t) \\
y_{r}(t) & =\mathbf{C}_{\mathbf{r}} x(t)+\mathbf{D}_{\mathbf{r}} u(t)
\end{aligned}
$$

where: $\mathbf{A} \in \mathbb{R}^{n \times n}, \mathbf{A}_{\mathbf{r}} \in \mathbb{R}^{k \times k}, \mathbf{B} \in \mathbb{R}^{n \times p}, \mathbf{B}_{\mathbf{r}} \in \mathbb{R}^{k \times p}, \mathbf{C} \in \mathbb{R}^{m \times n}, \mathbf{C}_{\mathbf{r}} \in \mathbb{R}^{m \times k}, \mathbf{D} \in \mathbb{R}^{m \times p}$, $\mathbf{D}_{\mathbf{r}} \in \mathbb{R}^{m \times p}$.

The reduced model state vector $x_{r}$ is related with the primary model state vector $x$ by transformation $x_{r}=T x$. There are many algorithms that can be applied to determine the matrix $T[1,2,3,10,12,17,18,20,21,23,27,33,34,35]$.

Number of methods enable complex model reduction. For models containing less than $10^{4}$ of the state variables, the highest approximation accuracy (which assures a reduced model stability) is provided by group of methods based on Singular Value Decomposition (SVD) [1, 2, 13].

Contrary to the reduction, abstraction does not approximate the properties of primary (complex) model by its lower order approximation. Its purpose is to determine a new model of primary model properties. To determine the controllability of complex model, conditions have been determined under which controllability of the abstract model implies controllability of the primary model [22]. 


\section{Hierarchical model of the BP-1150 boiler evaporator}

\subsection{Steam boiler BP-1150}

BB-1150 and BP-1150 boilers included in the power units of $360 \mathrm{MW}$ are monotube boilers based on the SULZER structure. Water separation at the outlet of the evaporator takes place in the vertical separator. In case of 30-85\% charges, the unit works at a sliding pressure, whereas for higher charges, it works at a constant pressure. The BB-1150 and BP-1150 are one-way boilers, about $100[\mathrm{~m}]$ high. The BP-1150 boiler evaporator contains evaporating tubes of a vertical membrane type which constitute the furnace walls, separator, mixer, filter, circulation pump and the connecting tubes (Fig. 3).

\subsection{Structure of the hierarchical model of the BP-1150 boiler evaporator}

General diagram of BP-1150 boiler showing hierarchical model structure, with particular focus on the evaporator model is presented in Fig. 4 [28, 29]. At 3-rd level, the evaporator model is connected with the models of other boiler devices. Operator description of the unit named 'evaporator' is as follows:

$$
\left[P_{\text {sep }} H_{\text {sep }}\right]^{T}=\text { evaporator }\left(\left[M_{s t} q^{\sim} M_{s a} M_{f w} h_{f w}\right]^{T}\right) .
$$

The evaporator model includes models of the following subsystems: evaporating tubes, separator, mixer, filter, circulation pump, steam attemperators supply node, and connecting tubes. Individual evaporator subsystems' models may be described by indicating input vectors $U_{2}^{i}$, output vectors $Y_{2}^{i}$ and by determining operators describing individual subsystems $F_{2}^{i}$ :

Evaporating tubes:

$$
\left[\begin{array}{lll}
h_{\text {sep }} & M_{\text {sep }} P_{w}
\end{array}\right]^{T}=\text { evaporator.evaporating_tubes }\left(\left[M_{w} q^{\sim} h_{w} P_{\text {sep }}\right]^{T}\right)
$$

Separator:

$$
\left[\begin{array}{ll}
P_{\text {sep }} H_{\text {sep }}
\end{array}\right]^{T}=\text { evaporator.separator }\left(\left[M_{\min } M_{\text {sep }} h_{\text {sep }} M_{s t}\right]^{T}\right)
$$

Mixer:

$$
\left[M_{\text {min }} h_{\text {mix }}\right]^{T}=\text { evaporator.mixer }\left(\left[M_{c p} M_{f w} h_{f w} P_{s e p}\right]^{T}\right)
$$

Filter:

$$
h_{f}=\text { evaporator.filter }\left(\left[M_{c p} h_{f i n}\right]^{T}\right)
$$

Circulation pump:

$$
M_{c p}=\text { evaporator.pump }\left(\left[P_{\text {sep }} P_{w} H_{\text {sep }} M_{\text {min }}\right]^{T}\right)
$$

Steam attemperators supply node:

$$
M_{w}=\text { evaporator.attemperators_node }\left(\left[M_{c p} M_{s a}\right]^{T}\right)
$$


Connecting tubes:

$h_{\text {fin }}=$ evaporator.connecting_tubes ${ }_{1}\left(h_{\text {mix }}\right)$

$h_{w}=$ evaporator.connecting_tubes $2\left(h_{f}\right)$.

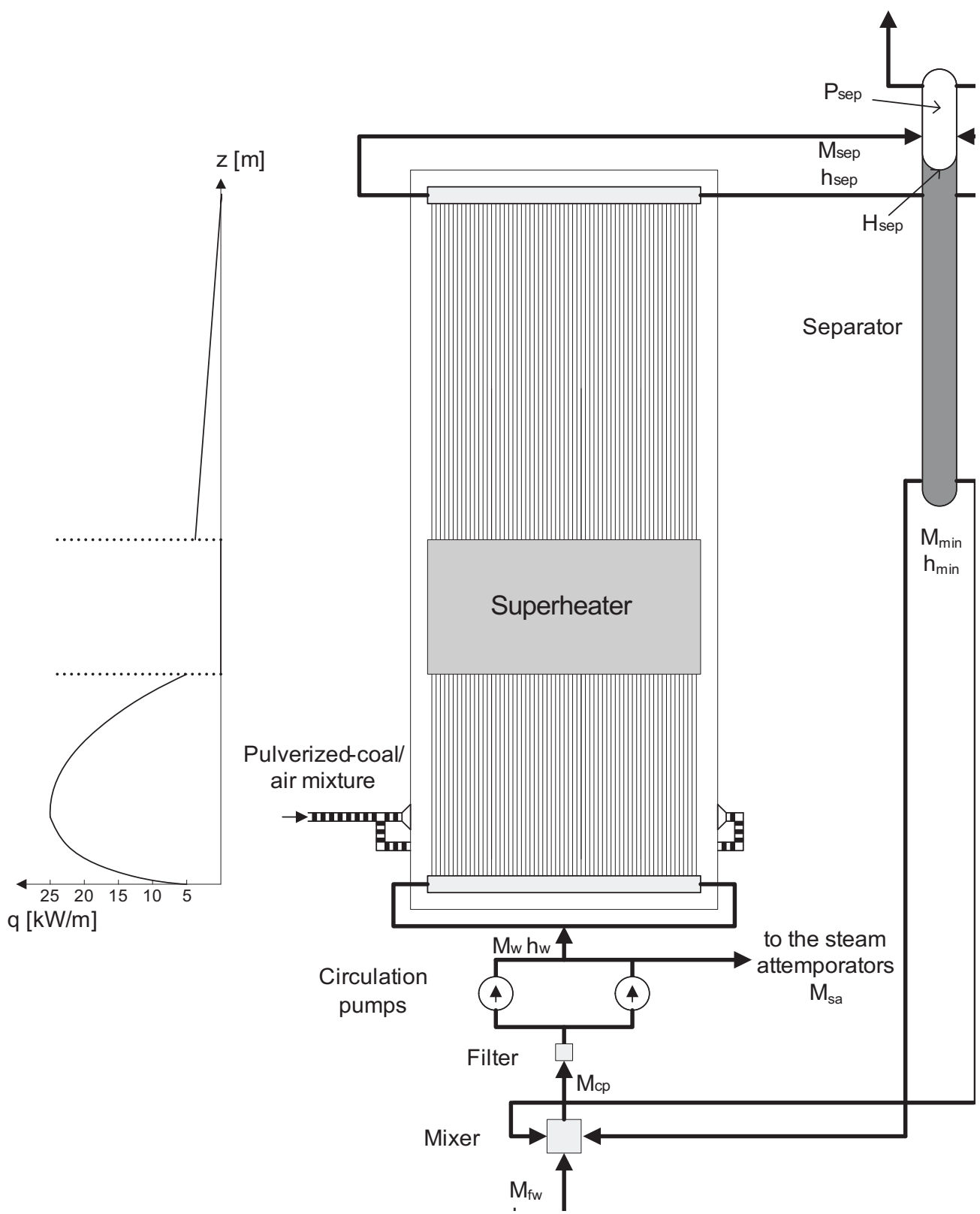

Figure 3. Simplified diagram of the BP-1150 boiler evaporator 


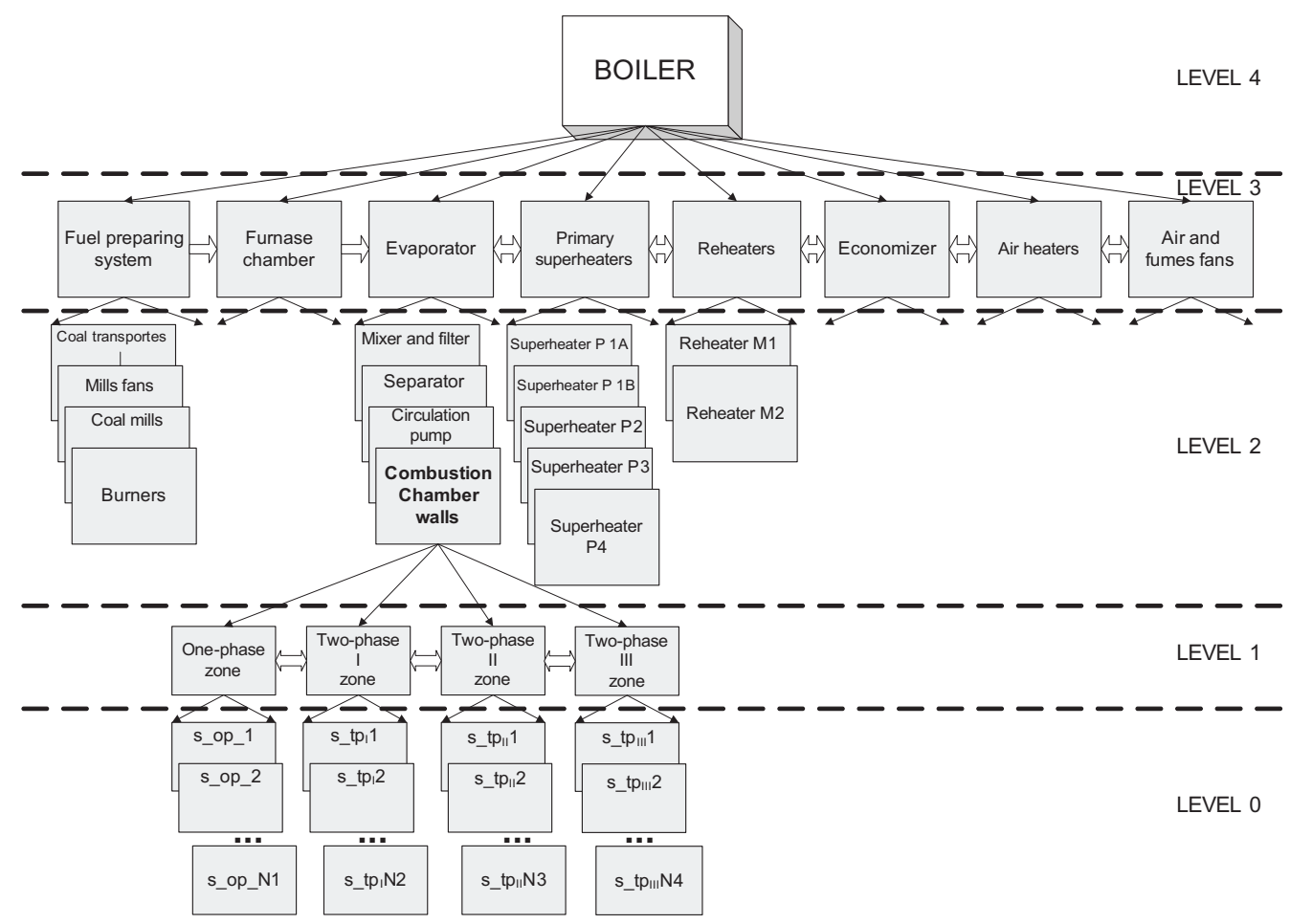

Figure 4. Hierarchical structure of the BP-1150 boiler model

Connections of subsystems models of BP-1150 boiler evaporator model are presented in form of a block diagram, in Figure 5.

The following BP-1150 boiler evaporator model subsystems: separator, mixer, filter, circulation pump, connecting tubes do not include lower level subsystems. The evaporating tubes model includes four following subsystems: one-phase zone, two-phase zone I, two-phase zone II, and two-phase zone III (these are the first level subsystems):

- one-phase zone extends from the inlet of the evaporating tubes to the section where volume boiling of water begins (water enthalpy reaches value $h^{\prime}(P)$ ) - section of $Z_{I}$ coordinate,

- two-phase zone I with high heat charge and intensive steam generation, extending from the $Z_{I}$ coordinate section to the place where the evaporating tubes are covered by the wall superheater (level $36.5 \mathrm{~m}$ ),

- two-phase zone II with zero heat charge, due to the cover by the wall superheater,

- two-phase zone III with insignificant heat charge and smaller flow velocity (evaporating tubes of larger diameter). 
The border section between one-phase and two-phase zones has got changeable $Z_{I}$ location and moves with $U_{I}$ velocity. The structure of evaporating tubes model of BP1150 boiler evaporator in form of block diagram, is presented in Figure 6.

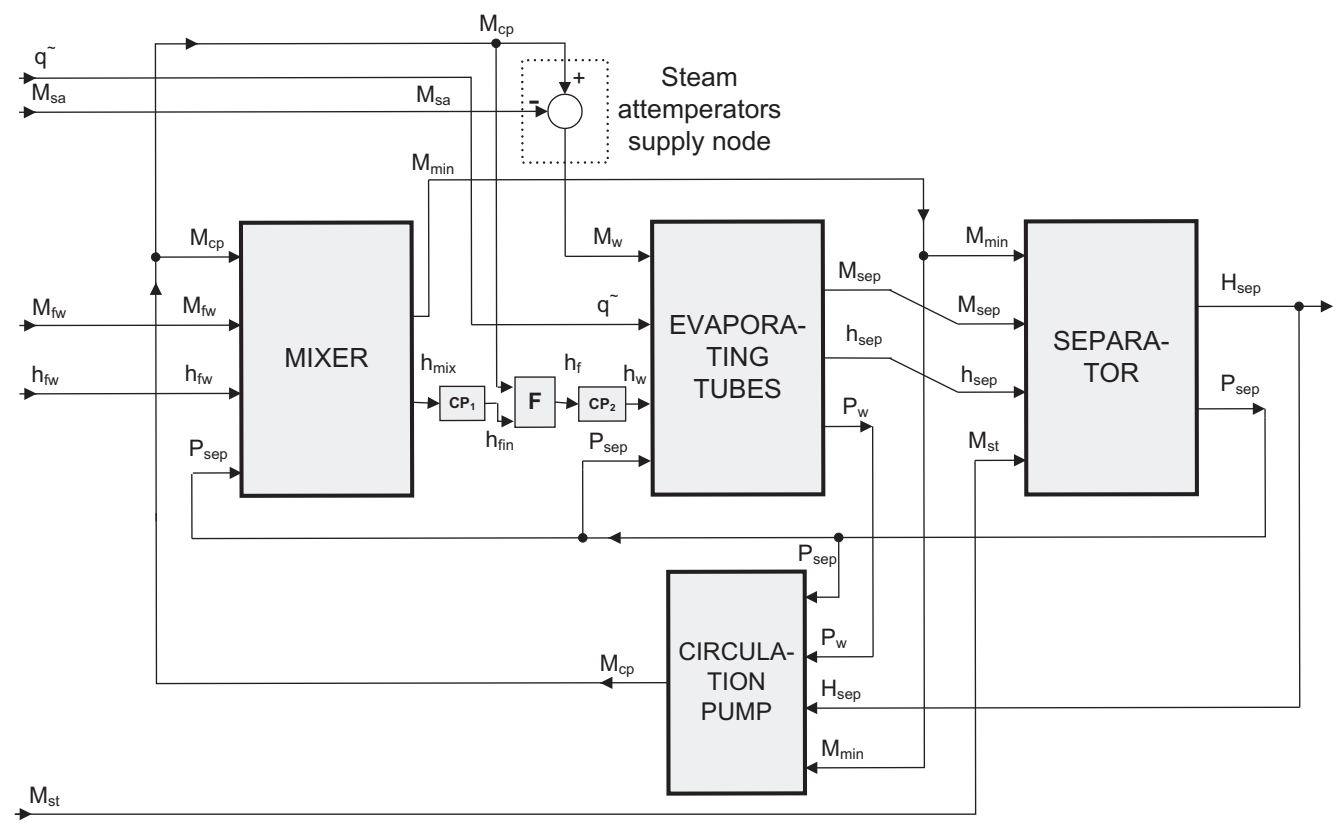

Figure 5. BP-1150 boiler evaporator model structure $\left(\mathrm{CP}_{1}, \mathrm{CP}_{2}\right.$ - connecting pipelines, $\mathrm{F}$ - filter $)$

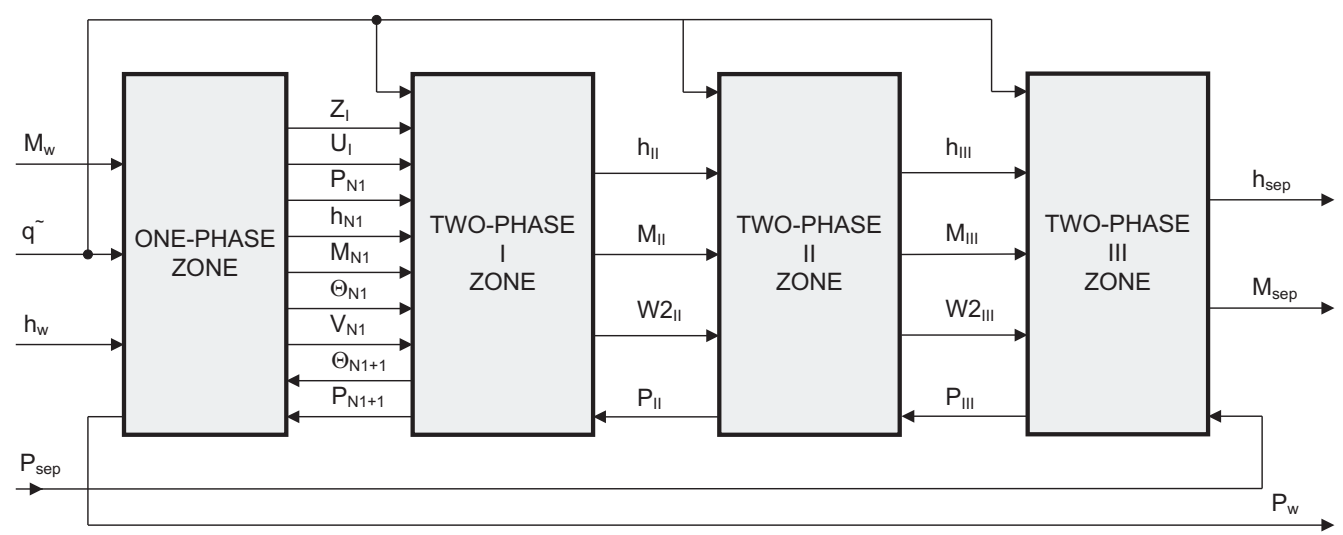

Figure 6. Evaporating tubes model structure of BP-1150 boiler evaporator 
Description of individual subsystem models of the evaporating tubes is as follows:

One-phase zone:

$\left[Z_{I} U_{I} P_{N 1} h_{N 1} M_{N 1} \Theta_{N 1} V_{N 1} P_{w}\right]^{T}=$ evaporator.evaporating_tubes.

$$
\text { .one-phase }\left(\left[M_{w} q^{\sim} h_{w} \Theta_{N 1+1} P_{N 1+1}\right]^{T}\right)
$$

Two-phase zone I:

$\left[h_{I I} M_{I I} W 2_{I I} \Theta_{N 1+1} P_{N 1+1}\right]^{T}=$ evaporator.evaporating_tubes.

$$
\text { .two-phase } \mathrm{I}\left(\left[q^{\sim} Z_{I} U_{I} P_{N 1} h_{N 1} M_{N 1} \Theta_{N 1} V_{N 1} P_{I I}\right]^{T}\right)
$$

Two-phase zone II:

$$
\begin{array}{r}
{\left[h_{I I I} M_{I I I} W 2_{I I I} P_{I I}\right]^{T}=\text { evaporator.evaporating_tubes. }} \\
\text {.two-phase } \operatorname{II}\left(\left[q \sim h_{I I} M_{I I} W 2_{I I} P_{I I I}\right]^{T}\right)
\end{array}
$$

Two-phase zone III:

$$
\begin{gathered}
{\left[h_{\text {sep }} M_{\text {sep }} P_{I I I}\right]^{T}=\text { evaporator.evaporating_tubes. }} \\
\text {.two-phase III }\left(\left[q^{\sim} h_{I I I} M_{I I I} W 2_{I I I} P_{\text {sep }}\right]^{T}\right) .
\end{gathered}
$$

At level no. 1 of the BP-1150 boiler model there are models of individual zones which have been determined by various kind of working medium flow in evaporating tubes. Individual subsystems of evaporating tubes are distributed parameter systems along the length and along the radius and circumference of the tubes, as well as along the connecting blades, creating the furnace walls. In the evaporator model with lumped parameters, the individual zones of the evaporating tubes have been divided along their length into large number of finite elements. Dimensions of finite elements are chosen to describe them by ordinary differential equations, as the systems with lumped parameters.

Particular feature of the one-phase and the first part of the two-phase zone models is shifting of the boundary section $Z_{I}$ with velocity $U_{I}$ (section with boiling water). For this reason, all finite elements of the one-phase zone model and first part of the two-phase zone model have got changeable dimensions and move along the evaporating tubes. For linearized models analyzed in the paper, displacements of the boundary section $Z_{I}$ are inconsiderable, so it is assumed that $Z_{I}=$ const and finite elements have got constant dimensions [28]. Exemplary model structure for second part of the two-phase zone is presented in Figure 7.

\subsection{Mathematical models of the evaporator subsystems}

\subsubsection{Evaporating tubes}

To describe phenomena in evaporating tubes, single tube with medium mass flow of working medium and average heat charge is considered (there are 1344 parallel tubes in the evaporator). Inner diameter of the tube is $0.02 \mathrm{~m}$ and its length is about $100 \mathrm{~m}$. So it is possible to analyze the evaporating tubes as a system with distributed parameters along the length (one-dimensional distributed parameter system). Balance equations describing 


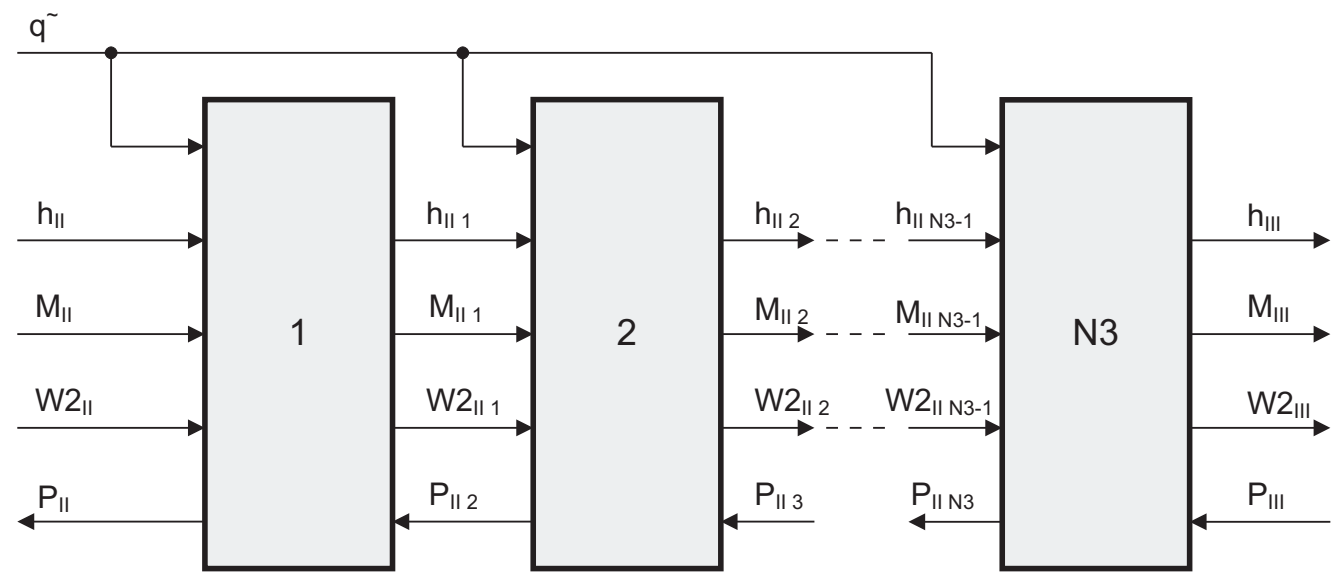

Figure 7. Two-phase zone II model structure

water flow in one-phase zone are as follows (balance of mass, energy and impulse of working medium) [28]:

$$
\begin{gathered}
\frac{\partial M}{\partial z}=-\frac{\partial \rho}{\partial t} \\
\rho \frac{\partial h}{\partial t}+M \frac{\partial h}{\partial z}-\frac{\partial P}{\partial t}-\frac{M}{\rho} \frac{\partial P}{\partial z}=q_{d}^{\prime} \\
\frac{\partial M}{\partial t}+2 \frac{M}{\rho} \frac{\partial M}{\partial z}-\frac{M^{2}}{\rho^{2}} \frac{\partial \rho}{\partial z}+\rho g \cos \beta+10^{3} \frac{\partial P}{\partial z}+f \frac{M^{2}}{\rho}=0
\end{gathered}
$$

where: $M=M(z, t)=\rho w, \rho=\rho(z, t)=\rho(P, h), h=h(z, t), P=P(z, t), \vartheta=\vartheta(P, h)$, $\alpha=\alpha(P, h, M, q), q_{d}^{\prime}=\frac{4\left(\Theta_{i n}-\vartheta\right) \alpha}{d}-$ the heat flux related to volume unit of working medium.

For one-dimensional two-phase flow, balans equations are as follows:

$$
\begin{gathered}
\frac{\partial M}{\partial z}=-\frac{\partial \rho}{\partial t} \\
\rho \frac{\partial h}{\partial t}+M \frac{\partial h}{\partial z}-\frac{\partial P}{\partial t}-\frac{M}{\rho} \frac{\partial P}{\partial z}=q_{d}^{\prime}-\frac{\partial}{\partial z}\left[M\left(\frac{w_{2}}{w}-1\right)\left(h-h^{\prime}\right)\right] \\
\frac{\partial M}{\partial t}+2 \frac{M}{\rho} \frac{\partial M}{\partial z}-\frac{M^{2}}{\rho^{2}} \frac{\partial \rho}{\partial z}+\rho g \cos \beta+10^{3} \frac{\partial P}{\partial z}+f \frac{M^{2}}{\rho}=0
\end{gathered}
$$


where: $M=M(z, t)=\rho w, \rho=\rho(z, t)=\rho(P, h), h=h(z, t), P=P(z, t), c=\frac{h-h^{\prime}}{h_{21}}$, $\frac{w_{2}}{w}=\frac{1}{c+\frac{1-c}{S}}, S=f(M, P, h)$.

Lumped parametr model is obtained by division evaporating tube into several finite elements. Individual finite elements have got small dimensions to constitute dynamic systems with lumped parameters (described by odrinary differential equations) (Fig. 8).

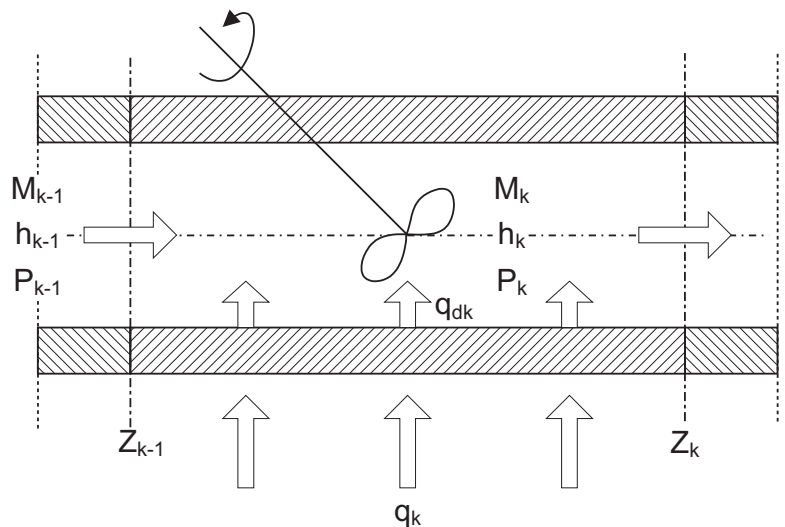

Figure 8. Finite element of the evaporating tube model

Balance equations describing water flow in $k$-th finite element of the one-phase zone have got the following form:

$$
\begin{gathered}
\Delta Z_{k}\left(\frac{\partial \rho}{\partial P}\right)_{k} \frac{\mathrm{d} P_{k}}{\mathrm{~d} t}+\Delta Z_{k}\left(\frac{\partial \rho}{\partial h}\right)_{k} \frac{\mathrm{d} h_{k}}{\mathrm{~d} t}=M_{k-1}-M_{k} \\
\Delta Z_{k} \rho_{k} \frac{\mathrm{d} h_{k}}{\mathrm{~d} t}-\Delta Z_{k} \frac{\mathrm{d} P_{k}}{\mathrm{~d} t}=M_{k-1}\left(h_{k-1}-h_{k}\right)- \\
\quad-\frac{M_{k-1}}{\rho_{k-1}}\left(P_{k-1}-P_{k}\right)+\frac{q_{d_{k}}}{A} \Delta Z_{k} \\
\Delta Z_{k} \rho_{k} \frac{\mathrm{d} M_{k}}{\mathrm{~d} t}=\frac{M_{k-1}^{2}}{\rho_{k-1}}-\frac{M_{k}^{2}}{\rho_{k}}- \\
-\Delta Z_{k} \rho_{k} g \cos \beta-\left(P_{k+1}-P_{k}\right) 10^{3}-\Delta Z_{k} f_{k} \frac{M_{k}^{2}}{\rho_{k}}
\end{gathered}
$$

where: $q_{i n_{k}}=\sum_{i=1}^{N_{\alpha}} \frac{\pi d}{N_{\alpha}} \alpha_{k}\left(\Theta_{k}^{i, 1}-\vartheta_{k}\right)$,

whereas for $k$-th two-phase zone section:

$$
\Delta Z_{k}\left(\frac{\partial \rho}{\partial P}\right)_{k} \frac{\mathrm{d} P_{k}}{\mathrm{~d} t}+\Delta Z_{k}\left(\frac{\partial \rho}{\partial h}\right)_{k} \frac{\mathrm{d} h_{k}}{\mathrm{~d} t}=M_{k-1}-M_{k}
$$




$$
\begin{aligned}
\Delta Z_{k} \rho_{k} \frac{\mathrm{d} h_{k}}{\mathrm{~d} t}-\Delta Z_{k} \frac{\mathrm{d} P_{k}}{\mathrm{~d} t}=M_{k-1}\left(h_{k-1}-h_{k}\right)-\frac{M_{k-1}}{\rho_{k-1}}\left(P_{k-1}-P_{k}\right)+ \\
+\frac{q_{d_{k}}}{A} \Delta Z_{k}+M_{k-1}\left[\left(\frac{w_{2}}{w}\right)_{k-1}-1\right]\left(h_{k-1}-h^{\prime}\right)- \\
-M_{k}\left[\left(\frac{w_{2}}{w}\right)_{k}-1\right]\left(h_{k}-h^{\prime}\right) \\
\Delta Z_{k} \rho_{k} \frac{\mathrm{d} M_{k}}{\mathrm{~d} t}=\frac{M_{k-1}^{2}}{\rho_{k-1}}-\frac{M_{k}^{2}}{\rho_{k}}-\Delta Z_{k} \rho_{k} g \cos \beta- \\
-\left(P_{k+1}-P_{k}\right) 10^{3}-\Delta Z_{k} f_{k} \frac{M_{k}^{2}}{\rho_{k}} .
\end{aligned}
$$

Working medium parameters in the two-phase zone are pressure $P$ and enthalpy $h$ functions:

$$
\begin{gathered}
\rho=f(P, h)=\frac{1}{(1-c) v^{\prime}+c v^{\prime \prime}}=\frac{h_{21}}{v_{21}} \frac{1}{h-\frac{h^{\prime} v^{\prime \prime}-h^{\prime \prime} v^{\prime}}{v_{21}}} \\
h_{21}=h^{\prime \prime}-h^{\prime}, v_{21}=v^{\prime \prime}-v^{\prime}, c=\frac{h-h^{\prime}}{h_{21}} \\
h^{\prime}=h^{\prime}(P), h^{\prime \prime}=h^{\prime \prime}(P),\left(\frac{\partial \rho}{\partial h}\right)_{k}=-\frac{\rho_{k}^{2} v_{21}}{h_{21}} \\
\left(\frac{\partial \rho}{\partial P}\right)_{k}=\frac{\rho_{k} v_{21}}{h_{21}} \frac{\mathrm{d}}{\mathrm{d} P}\left(\frac{h_{21}}{v_{21}}\right)+\frac{\rho_{k}^{2} v_{21}}{h_{21}} \frac{\mathrm{d}}{\mathrm{d} P}\left(\frac{h^{\prime} v^{\prime \prime}-h^{\prime \prime} v^{\prime}}{v_{21}}\right) .
\end{gathered}
$$

Equations (10) and (11) should be completed with the relations describing the heat transfer, from the furnace chamber, along the radius and the tube wall cimcurference, as well as along the connecting blade.

Tube wall:

$$
\begin{aligned}
\frac{\mathrm{d} \Theta_{k}^{i, j}}{\mathrm{~d} t} & =\frac{4 \lambda_{t w}}{\rho_{t w} c_{t w} \Delta} \frac{\left[(d+2 j \Delta)\left(\Theta_{k}^{i, j+1}-\Theta_{k}^{i, j}\right)-(d+2(j-1) \Delta)\left(\Theta_{k}^{i, j}-\Theta_{k}^{i, j-1}\right)\right]}{\left[(d+2 j \Delta)^{2}-(d+2(j-1) \Delta)^{2}\right]}+ \\
& +\frac{4 N_{\alpha}^{2} \Delta \lambda_{t w}}{\pi^{2} \rho_{t w} c_{t w}[d+(2 j-1) \Delta]} \frac{\Theta_{k}^{i-1, j}-2 \Theta_{k}^{i, j}+\Theta_{k}^{i+1, j}}{\left[(d+2 j \Delta)^{2}-(d+2(j-1) \Delta)^{2}\right]}
\end{aligned}
$$




$$
\begin{aligned}
\frac{\mathrm{d} \Theta_{k}^{i, 1}}{\mathrm{~d} t} & =\frac{4(d+2 \Delta) \lambda_{t w}}{\rho_{t w} c_{t w} \Delta\left[(d+2 \Delta)^{2}-d^{2}\right]}\left(\Theta_{k}^{i, 2}-\Theta_{k}^{i, 1}\right)- \\
& -\frac{4 d \alpha_{k}}{\rho_{t w} c_{t w}\left[(d+2 \Delta)^{2}-d^{2}\right]}\left(\Theta_{k}^{i, 1}-\vartheta_{k}\right)+ \\
& +\frac{4 N_{\alpha}^{2} \Delta \lambda_{t w}}{\pi^{2} \rho_{t w} c_{t w}(d+\Delta)\left[(d+2 \Delta)^{2}-d^{2}\right]}\left(\Theta_{k}^{i-1,1}-2 \Theta_{k}^{i, 1}+\Theta_{k}^{i+1,1}\right) \\
\frac{\mathrm{d} \Theta_{k}^{i, N_{t w}}}{\mathrm{~d} t}= & \frac{4 N_{\alpha}}{\pi \rho_{t w} c_{t w}\left[D^{2}-(D-2 \Delta)^{2}\right]} \frac{D}{D+L_{b l}} \frac{1}{\frac{N_{\alpha}}{2}-1} q+ \\
+ & \frac{4 \lambda_{t w}(D-2 \Delta)}{\rho_{t w} c_{t w} \Delta\left[D^{2}-(D-2 \Delta)^{2}\right]}\left(\Theta_{k}^{i, N_{t w}-1}-\Theta_{k}^{i, N_{t w}}\right)+ \\
+ & \frac{4 N_{\alpha}^{2} \Delta \lambda_{t w}}{\pi^{2} \rho_{t w} c_{t w}(D-\Delta)\left[D^{2}-(D-2 \Delta)^{2}\right]}\left(\Theta_{k}^{i-1, N_{t w}}-2 \Theta_{k}^{i, N_{t w}}+\Theta_{k}^{i+1, N_{t w}}\right)
\end{aligned}
$$

where: $\Delta=\frac{D-d}{2 N_{t w}}, N_{t w}$ - number of layers in the tube wall, $D, d$ - inner and outer tube diameter, $N_{\alpha}$ - number of elements in the tube wall cimcurference, $L_{b l}$ - length of the connecting blade.

Connecting blade:

$$
\begin{aligned}
\frac{\mathrm{d} \Theta_{b l k}^{l}}{\mathrm{~d} t}= & \frac{\lambda_{b l}}{\Delta_{b l} \rho_{b l} c_{b l}}\left[\Theta_{b l k}^{l-1}-2 \Theta_{b l k}^{l}+\Theta_{b l k}^{l+1}\right]+\frac{L_{b l}}{D+L_{b l}} \frac{1}{N_{b l} \delta_{b l} \Delta_{b l} \rho_{b l} c_{b l}} q_{k} \\
\frac{\mathrm{d} \Theta_{b l k}^{1}}{\mathrm{~d} t}= & \frac{\lambda_{b l}}{\Delta_{b l}^{2} \rho_{b l} c_{b l}}\left(\Theta_{k}^{i_{l l}, N_{t w}}-\Theta_{b l k}^{1}\right)+\frac{\lambda_{b l}}{\Delta_{b l}^{2} \rho_{b l} c_{b l}}\left(\Theta_{b l k}^{2}-\Theta_{b l k}^{1}\right)+ \\
& +\frac{L_{b l}}{D+L_{b l}} \frac{1}{N_{b l} \delta_{b l} \Delta_{b l} \rho_{b l} c_{b l}} q_{k} \\
\frac{\mathrm{d} \Theta_{b l k}^{N_{b l}}}{\mathrm{~d} t} & =\frac{\lambda_{b l}}{\Delta_{b l}^{2} \rho_{b l} c_{b l}}\left(\Theta_{b l k}^{N_{b l}-1}-\Theta_{b l k}^{N_{b l}}\right)+\frac{L_{b l}}{D+L_{b l}} \frac{1}{N_{b l} \delta_{b l} \Delta_{b l} \rho_{b l} c_{b l}} q_{k}
\end{aligned}
$$

where: $\delta_{b l}$ - thickness of the connecting blade, $\Delta_{b l}=\frac{L_{b l}}{N_{b l}}$.

Diagram of the tube wall and connecting blade digitizing is presented in Fig. 9.

\subsubsection{Separator}

The role of separator in once-through steam boilers by SULZER is separation of water from the steam-water mixture leaving the evaporating tubes. Separator constitutes cylindrical vertical container, with length of $L_{s e p} \approx 34 \mathrm{~m}$., inner diameter $d=0.8 \mathrm{~m}$. and 


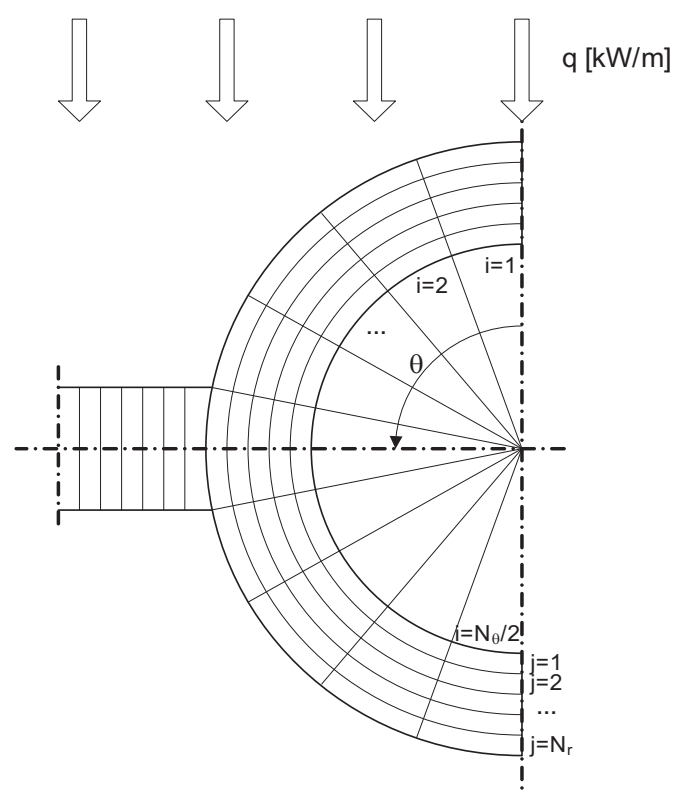

Figure 9. Digitizing of the tube wall, including irregularities of the heat stream along the tube circumference

external diameter $D=0.94 \mathrm{~m}$. Steam-water mixture is supplied to the top part of the separator with the velocity about $16 \mathrm{~m} / \mathrm{s}$, through tangent connecting tubes. Via the bottom connecting tubes, also tangent, water is supplied to the mixer, with velocity about $2.5 \mathrm{~m} / \mathrm{s}$. The mechanism of water separation in the field of centrifugal forces in the separator is very complex process, dependent on series of physical, construction and exploitation parameters.

Separator is the system with four input values: $M_{s t}, M_{\text {sep }}, h_{\text {sep }}, M_{m i n}$, and two output values: $P_{\text {sep }}, H_{\text {sep }}$. A lumped parameters model of the separator is used in this paper, based on balance of mass and energy of the working medium, as well as the heat energy in the steel thick wall [28].

The balances of mass and energy of the working medium in the separator are as follows:

$$
\left[\begin{array}{ll}
a & b \\
c & d
\end{array}\right]\left[\begin{array}{c}
\frac{\mathrm{d} P_{\text {sep }}}{\mathrm{d} t} \\
\frac{\mathrm{d} H_{\text {sep }}}{\mathrm{d} t}
\end{array}\right]=\left[\begin{array}{c}
M_{\text {sep }}^{*}-M_{p}^{*}-M_{k o n d}^{*} \\
M_{s e p}^{*} h_{s e p}^{*}-M_{p}^{*} h^{\prime \prime}-M_{k o n d}^{*} h^{\prime}-\pi d \alpha L_{\text {sep }}\left(\vartheta-\Theta_{1}\right)
\end{array}\right]
$$

where: $a=A_{z} H_{\text {sep }} \frac{\mathrm{d} \rho^{\prime}}{\mathrm{d} P}+\left(V-A_{z} H_{\text {sep }}\right) \frac{\mathrm{d} \rho^{\prime \prime}}{\mathrm{d} P}, b=A_{z}\left(\rho^{\prime}-\rho^{\prime \prime}\right)$,

$c=A_{z} H_{\text {sep }} \frac{\mathrm{d}\left(\rho^{\prime} h^{\prime}\right)}{\mathrm{d} P}+\left(V-A_{z} H_{\text {sep }}\right) \frac{\mathrm{d}\left(\rho^{\prime \prime} h^{\prime \prime}\right)}{\mathrm{d} P}, d=A_{z}\left(\rho^{\prime} h^{\prime}-\rho^{\prime \prime} h^{\prime \prime}\right)$. 
The separator's steel wall is described by the following state vector:

$$
\frac{\mathrm{d}}{\mathrm{d} t}\left[\begin{array}{c}
\Theta_{1} \\
\vdots \\
\Theta_{k} \\
\vdots \\
\Theta_{N}
\end{array}\right]=\left[\begin{array}{c}
4 \frac{d \alpha \Delta\left(\vartheta-\Theta_{1}\right)-(d+2 \Delta) \lambda\left(\Theta_{1}-\Theta_{2}\right)}{\left((d+2 \Delta)^{2}-d^{2}\right) \rho_{s t} c_{s t} \Delta} \\
\vdots \\
4 \frac{(d+2(k-1) \Delta) \lambda\left(\Theta_{k-1}-\Theta_{k}\right)-(d+2 k \Delta) \lambda\left(\Theta_{k}-\Theta_{k+1}\right)}{\left((d+2 k \Delta)^{2}-(d+2(k-1) \Delta)^{2}\right) \rho_{s t} c_{s t} \Delta} \\
\vdots \\
4 \frac{(d+2(N-1) \Delta) \lambda\left(\Theta_{N-1}-\Theta_{N}\right)}{\left(D^{2}-(D-2 \Delta)\right) \rho_{s t} c_{s t} \Delta}
\end{array}\right] .
$$

Calculations indicate that the separator is unstable subsystem. This instability is related with pressure dependency of dry steam and the boiling water parameters. The pole of the separator model (Fig. 10), which implies instability, depends strongly on the boiler load. The pole value is greatest for $100 \%$ load [28]. Instability of the separator influences instability of the whole evaporator.

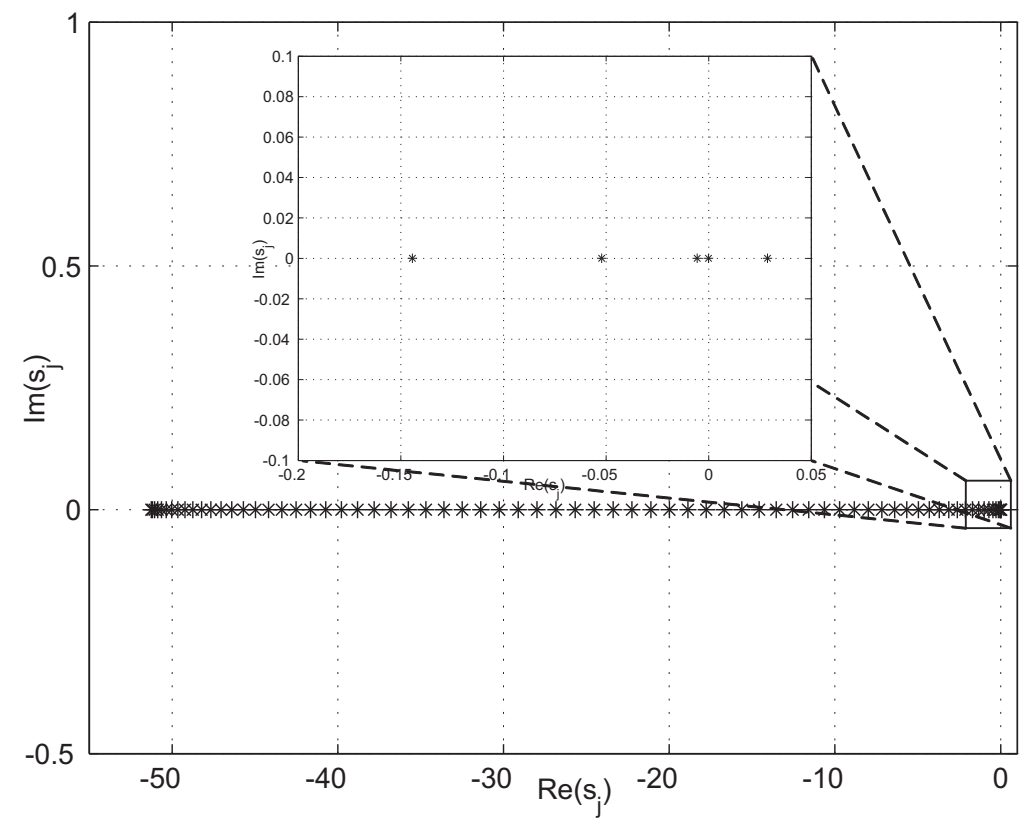

Figure 10. The separator's eigenvalues

Mathematical models of the remaining evaporator subsystems (mixer, filter, connecting tubes and circulation pump) have been described in [28]. They are postponed here due to their simplicity. 
All obtained models were implemented in MATLAB/Simulink environment. On the basis of these models, linearized models were developed at a given working point of a BP-1150 boiler (e.g. at 100\% load).

\section{Evaporator model reduction at individual hierarchy levels}

Once-through evaporator mathematical model (elaborated on the basis of the liquid mechanics and thermodynamics rules for mass, energy and momentum balance equations) has been characterized with a high degree of complexity. It is significantly difficult to be used directly to programme methods of dynamic properties analysis as well as to synthesize control algorithms. The basic problem becomes then the choice of proper reduction methods, so that the model complexity is limited, while dynamic features (significant at a given hierarchy level) are preserved.

\subsection{Scopes of evaporator models adequacy at individual hierarchy levels}

The elaborated evaporator models have been analyzed for a wide frequency range (up to $1000[\mathrm{rad} / \mathrm{s}]$ ), however adequacy scope of the primary model is limited due to the following simplifying assumptions:

- the steam-water mixture is in a state of thermodynamic balance (this assumption is valid for $\omega<100[\mathrm{rad} / \mathrm{s}])$,

- the simplification of heat energy transport along the pipe wall model (applied model assures adequacy for frequencies $\omega \leqslant 20[\mathrm{rad} / \mathrm{s}])$,

- applied density of space variable digitizing assures adequacy up to about 20 [rad/s] $[28,30]$.

We can assume, that adequacy scope of evaporator models is less or equal to $20[\mathrm{rad} / \mathrm{s}]$. Individual dynamic processes take place with various velocities and they can be placed in different frequency scopes:

- $\omega<0.2[\mathrm{rad} / \mathrm{s}]$

Within this frequency scope, there are dynamic processes related to the accumulation of heat in the pipe walls and in the working medium, as well as to the heat exchange between the pipe walls and the working medium. The limit frequency value for this scope results from the working medium particle flow time through the evaporating tubes $\left(\tau_{p} \approx 30 \mathrm{~s}\right) \omega=2 \pi / 30 \approx 0.2[\mathrm{rad} / \mathrm{s}]$. It means that there exist dynamic processes in this frequency scope which are longer than the time spent by the working medium particle in evaporating tubes and the mass transport processes are not noticeable.

- $2>\omega>0.2[\mathrm{rad} / \mathrm{s}]$

There are dynamic processes noticed in this frequency scope which are related to 
the working medium transport along the evaporating tubes, and also to the density and changes of working medium enthalpy. Maximum frequency for this scope results from the time spent by the particle of the working medium in the first part of evaporation zone (the shortest time spent by the particle of working medium in all parts of evaporating tubes) $(\tau \approx 3.0 \mathrm{~s}) \omega=2 \pi / 3 \approx 2[\mathrm{rad} / \mathrm{s}]$.

- $\omega>2[\mathrm{rad} / \mathrm{s}]$

There are phenomena in this frequency scope which are related to the transfer of the mass flow and pressure changes along the evaporating tubes. The pressure changes transfer time along the evaporating tubes is $\tau \approx 0.5$ [s]. Pressure changes, after being reflected from the other end of the evaporating tubes return after about 1 [s] which causes, that the first maximum in the frequency response induced by the pressure changes transfer, appears at the frequency $\omega=2 \pi / \tau \approx 6[\mathrm{rad} / \mathrm{s}]$.

Fig. 11 shows example of magnitude frequency response of the evaporating tubes for $\mathrm{BP}-1150$ boiler in order to illustrate the above defined frequency scopes.

Connecting of model reduction methods with evolution algorithms (which are aimed to determine optimal parameters for reduction methods) enables effective determining of reduced models for chosen approximation errors and adequacy scopes [25]. Obtained models may be useful not only for control systems design but also for simulation and analysis of the evaporator dynamic properties.

Due to the problem of stability of the reduced hierarchical model, it becomes necessary to implement the idea of approximation scope. This idea is close to adequacy scope and for the linear systems can be defined as frequency scope for which the reduced model correctly approximates properties of the primary model. Both terms are identical to frequency 20 [rad/s], for the evaporator subsystems models. Out of the range reduced model cannot be called adequate (primary model for this scope of frequency is inadequate), but correctly approximating the primary model features. Fig. 12 presents sample adequacy and approximation scopes for reduced models.

\subsection{Linearized models reduction methods}

Most of contemporarily used methods of linear models reduction can be divided into three groups $[1,2]$ :

- Methods based on SVD (Singular Value Decomposition), which use the balanced model realization theory.

- Krylov-based approximation methods, based on moment matching of the impulse response.

- Methods which connect the advantages of the both SVD and Krylov group.

Model reduction methods based on SVD have been commenced with Moore's works [20]. The concept of balanced model realization was a turning point in the field of dynamic linear models reduction. It constitutes an easy way to determine the dominating 


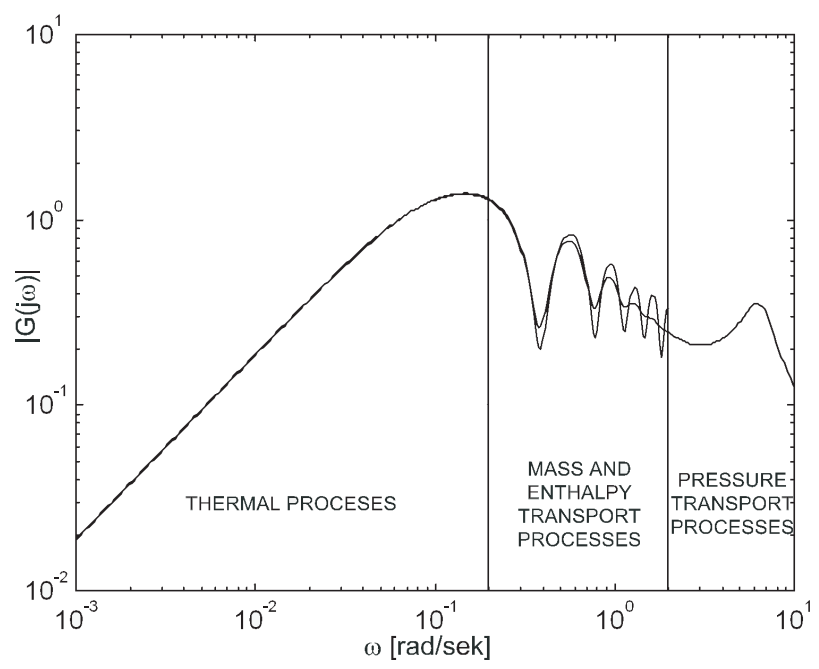

Figure 11. Mass flow magnitude frequency response at the outlet of evaporating tubes for heat stream imposing

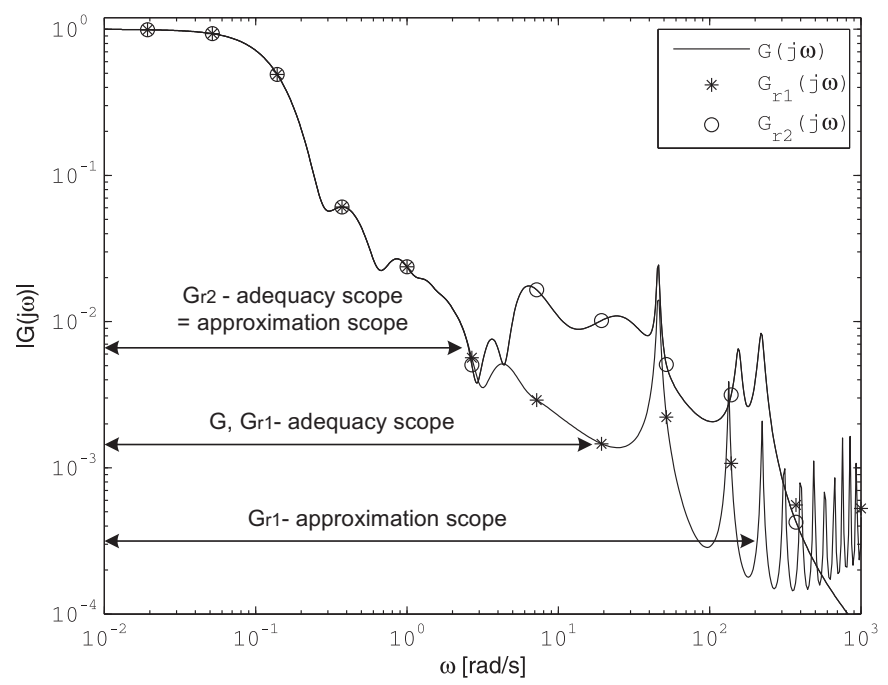

Figure 12. Adequacy and approximation scopes for reduced models

part of the model and reduction by 'cutting' the matrices describing dynamics of the model in state space (Balanced Truncation). The concept of model balanced realization became the basis for elaboration of BTA reduction method (Balanced Truncation Approximation) [20]. Main advantages of this reduction method is guarantee of reduced model stability and possibility to estimate the approximation error. This method was elaborated preliminarily for asymptotically stable linear models and became the basis for developing unstable models reduction methods [6]. Many modifications of BTA method 
have also been created to eliminate its defects e.g.: SPA (Singular Perturbation Approximation) $[6,19]$. This method significantly improves approximation of frequency responses for steady state and for low frequencies.

In 1984 an optimum reduction method was presented with regard to the Hankel norm (Hankel Norm Approximation) [10]. HNA method guarantees a double smaller maximum approximation error than BTA, however, it is loaded with higher calculation complexity of the algorithm.

More general than BTA metod are FW methods (Frequency Weighted), which introduce weight frequency functions $\left(W_{i}\right.$ - input weight function and $W_{o}$ - output weight function). Proper selection of the weight functions enables significant improvement of the model approximation results for a given frequency scope. The first method of this type was proposed by Enns in 1984 [7], however, the presented algorithm did not guarantee stability of reduced model for simultaneous application of both weight functions. This disadvantage was removed in algorithms proposed by Lin and Chiu and Wang [34].

Frequency weights are also possible to be introduced directly. Two methods can be distinguished: FD (Frequency Domain) [1, 2, 9, 25, 36], if gramians are determined in frequency domain and TLBT (Time Limited Balanced Truncation) $[1,2,9,14]$ for time domain.

The Balanced Stochastic Truncation - BST methods constitute a separate group of methods $[1,4,21]$, determining gramians from Riccatti equations. The methods enable determining reduced model of assumed relative approximation error. However, application of BST method is possible only for stable square models $(p=m)[1,21]$.

Disadvantage of methods based on SVD is considerable numerical complexity of the reduction algorithms. In spite of their unquestionable advantages, including first of all the reduced model stability, the methods are not practically used for models of state variables number higher than $10^{4}$. It is caused by a significant calculation complexity and impractical nature of gramians.

For systems with more than $10^{4}$ state variables the Krylov-based approximation methods are proposed $[1,2]$. They are characterized with smaller calculation complexity. Main disadvantage of these reduction methods are lack of stability guarantee of the reduced model (for significant part of the elaborated algorithms). These methods are usually characterized with less accurate approximation of the frequency response as compared with BT methods, especially with methods implementing frequency weighs [13].

One of the simplest reduction approach based on the method of moments is AWE method (Asymptotic Waveform Evaluation), proposed by Pillage and Rohrer in 1990 [24]. The method utilizes the fact that the transfer function of primary model, presented as asymptotic series expansion at two points $s=0$ and $s=\infty$ of the complex plane, can be well approximated with Pade polynomial. Direct determining of the moments values is, however, difficult because of numerical reasons. This limits significantly the usefulness of the method for reduction purposes [8].

Much better are algorithms basing on Krylow subspace determining [1, 5]. The methods require determining of orthonormal basis. Two algorithms are the most impor- 
tant: Arnoldi algorithm (modified Gram-Schmidt orthogonalization) and asymmetrical Lanczos algorithm.

The Krylov-SVD methods enable replacing a time consuming calculation of gramians from Lapunov equations by determining their approximation $[1,3,11,18,23])$. Similar principle was used for determining gramians decomposition with POD algorithm (Proper Orthogonal Decomposition) [15].

The most advantageous method $[16,25,32]$ for the reduction of the evaporator subsystems models is the FW method with output weight functions in the form of Butterworth low-pass filters, determined by two parameters: filter order $n_{f}$ and the limit frequency $\omega_{f}$. Number of calculations showed, that the choice of output filters parameters significantly influences the primary model approximation error. Criterion of approximation correctness should include the relative maximum error and the relative average square error $[25,26]$ :

$$
\begin{gathered}
\Delta_{3}=100 \max _{\omega_{i} \in\left(0-\omega_{\max }\right)}\left(\max _{x, y} \frac{\left|G_{(x, y)}\left(j \omega_{i}\right)-G_{r(x, y)}\left(j \omega_{i}\right)\right|}{\left|G_{(x, y)}\left(j \omega_{i}\right)\right|}\right)[\%] \\
\Delta_{6}=100 \sqrt{\frac{1}{p m N} \sum_{i=1}^{N} \sum_{x=1}^{p} \sum_{y=1}^{m}\left(\frac{\left|G_{(x, y)}\left(j \omega_{i}\right)-G_{r(x, y)}\left(j \omega_{i}\right)\right|}{\left|G_{(x, y)}\left(j \omega_{i}\right)\right|}\right)^{2}}[\%]
\end{gathered}
$$

where: $p$ - number of model inputs, $m$ - number of model outputs, $N$ - number of approximation points in frequency domain.

The approximation error is a function of weight filters parameters and includes a lot of local extremes while determining optimal parameters of weight filters, thus global optimization methods should be applied. To obtain an exact result it becomes necessary to apply hybrid algorithms, coupling global and local search algorithms. The evolutionary algorithms with variable population are used in this paper. Very good properties are obtained by application of two level hybrid algorithms [25].

\subsection{Reduction of the first level models}

The first level of the evaporator model includes one-phase and two-phase zones models which are a part of the evaporating tubes model. They include number of connected finite elements models, which are the 55-th order system each:

- One-phase zone - 150 finite elements,

- Two-phase zone I: - 50 finite elements,

- Two-phase zone II: - 75 finite elements,

- Two-phase zone III - 200 finite elements. 
The models obtained on the basis of digitizing and description of individual finite elements by ordinary differential equations are very complex and include a few thousand state variables. Models at a given hierarchy level include insignificant, sometimes very fast, dynamic processes. Moreover, mathematical models have got a defined adequacy scope due to accepted simplifying assumptions. A model reduction is therefore necessary in order to eliminate fast dynamic processes from the models.

The most favorable for the evaporator subsystems models reduction is FW method. Number of outputs is smaller than inputs, thus it is more advantageous to apply output weights. FW algorithm with output weights is then applied to reduce of all evaporator subsystems.

The approximation error is presented in Fig. 13 as a function of the reduced model order for individual evaporating tubes zones. To determine these curves the evolutionary algorithm minimizing average square relative approximation error $\left(\Delta_{6}\right)$ for the scope of frequencies $0-20[\mathrm{rad} / \mathrm{s}]$ is applied. It can be noticed, that for all evaporating tubes zones, the approximation error is reduced exponentially with the increase of the reduced model order. It is possible to determine the approximated order of reduced model for a given value of approximation error. Results of the first level subsystems reduction are presented in Table 1.
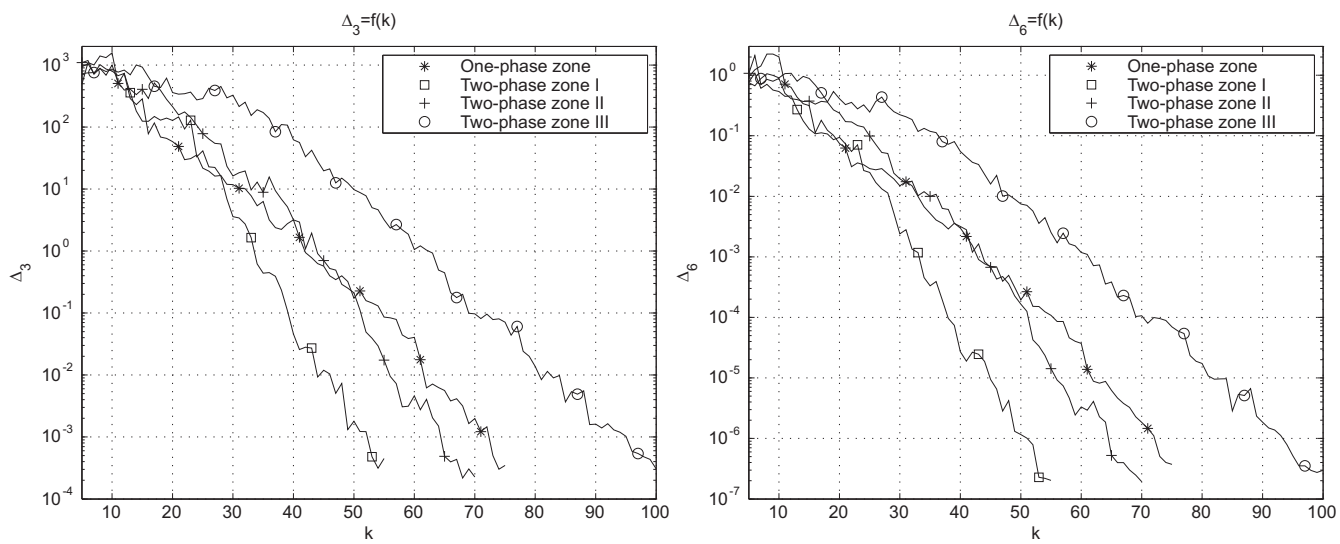

Figure 13. Model approximation errors of the reduced models

Fig. 14 includes a 'cloud' of eigenvalues for the following evaporating tube zones models: primary model and reduced models with approximation scopes $500[\mathrm{rad} / \mathrm{s}]$, $20[\mathrm{rad} / \mathrm{s}]$ and $2[\mathrm{rad} / \mathrm{s}]\left(\mathrm{OPZPM}, \mathrm{OPZRM}_{500}, \mathrm{OPZRM}_{20}, \mathrm{OPZRM}_{2}\right)$. The primary model is characterized with high stiffness (ratio of eigenvalues modules reaches the value of $10^{4}$ ), which causes significant difficulties followed from numerical solving of differential equations. In reduced models a significant stiffness restriction is obtained (e.g. for reduced model adequacy scope $20[\mathrm{rad} / \mathrm{s}]$, the ratio of eigenvalues modules does not exceed $10^{2}$ ). 
Table 1. The first level subsystems models reduction results

\begin{tabular}{|c|c|c|c|c|}
\hline & $\begin{array}{l}\text { One-phase } \\
\text { zone }\end{array}$ & $\begin{array}{l}\text { Two-phase } \\
\text { zone I }\end{array}$ & $\begin{array}{l}\text { Two-phase } \\
\text { zone II }\end{array}$ & $\begin{array}{l}\text { Two-phase } \\
\text { zone III }\end{array}$ \\
\hline Adequacy scope & \multicolumn{5}{|c|}{$\omega_{\max }=2[\mathrm{rad} / \mathrm{s}]$} \\
\hline Reduced model order & 18 & 13 & 17 & 22 \\
\hline$\Delta_{3}$ Approximation error & $6.8 \%$ & $4.9 \%$ & $7.6 \%$ & $9.0 \%$ \\
\hline$\Delta_{6}$ Approximation error & $1.1 \%$ & $0.4 \%$ & $1.1 \%$ & $1.1 \%$ \\
\hline Adequacy scope & \multicolumn{5}{|c|}{$\omega_{\max }=20[\mathrm{rad} / \mathrm{s}]$} \\
\hline Reduced model order & 33 & 29 & 35 & 52 \\
\hline$\Delta_{3}$ Approximation error & $7.8 \%$ & $8.2 \%$ & $8.9 \%$ & $7.7 \%$ \\
\hline$\Delta_{6}$ Approximation error & $1.1 \%$ & $0.7 \%$ & $1.1 \%$ & $0.6 \%$ \\
\hline Adequacy scope & \multicolumn{5}{|c|}{$\omega_{\max }=500[\mathrm{rad} / \mathrm{s}]$} \\
\hline Reduced model order & 68 & 78 & 113 & 242 \\
\hline$\Delta_{3}$ Approximation error & $6.8 \%$ & $9.8 \%$ & $7.9 \%$ & $7.3 \%$ \\
\hline$\Delta_{6}$ Approximation error & $0.7 \%$ & $0.6 \%$ & $0.7 \%$ & $0.3 \%$ \\
\hline
\end{tabular}
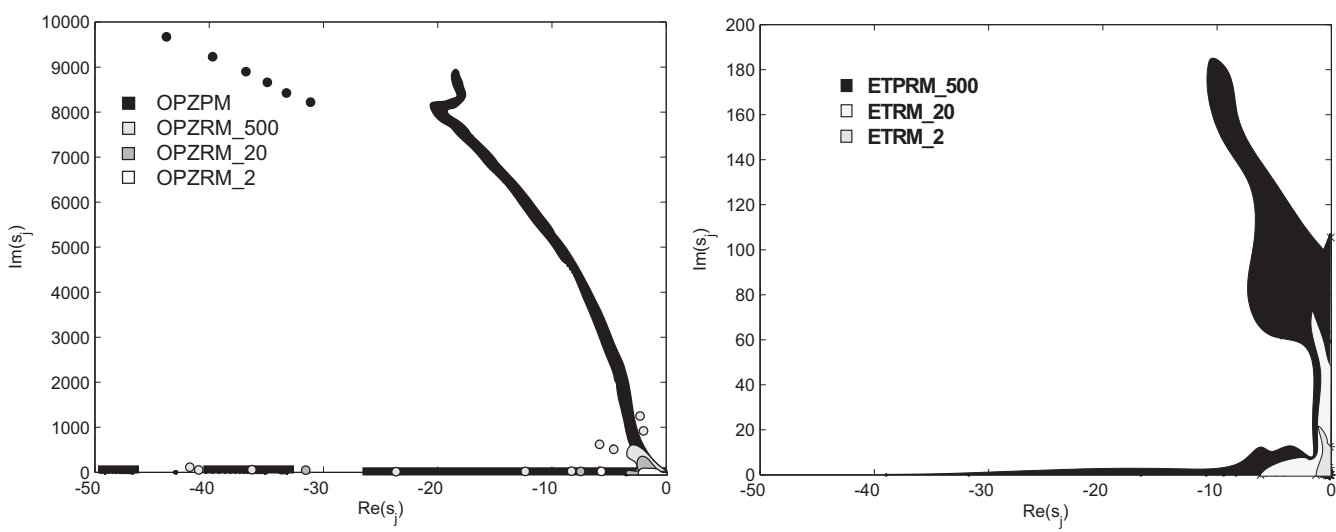

Figure 14. Eigenvalues of the one-phase zone and evaporating tubes models

\subsection{Reduction of the second hierarchy level models}

Evaporating tubes model (the second level of the evaporator model hierarchy) is obtained as a result of connecting the first level reduced subsystems models (one-phase zone and three parts of two-phase zone) (Fig. 15). Depending on accuracy of dynamics analysis of individual evaporating tubes parts, models of various adequacy level may be used. Maximum frequency for which reduced first level models remain adequate is $20[\mathrm{rad} / \mathrm{s}]$. 
As presented in Fig. 15, there are feedbacks in the model of evaporating tubes which result from the transfer of working medium pressure changes in the direction of the working medium flow and in the opposite direction. The feedbacks interact very weakly at low frequencies; for high frequencies the gain in the feedback loop exceeds 10 (Fig. 16).

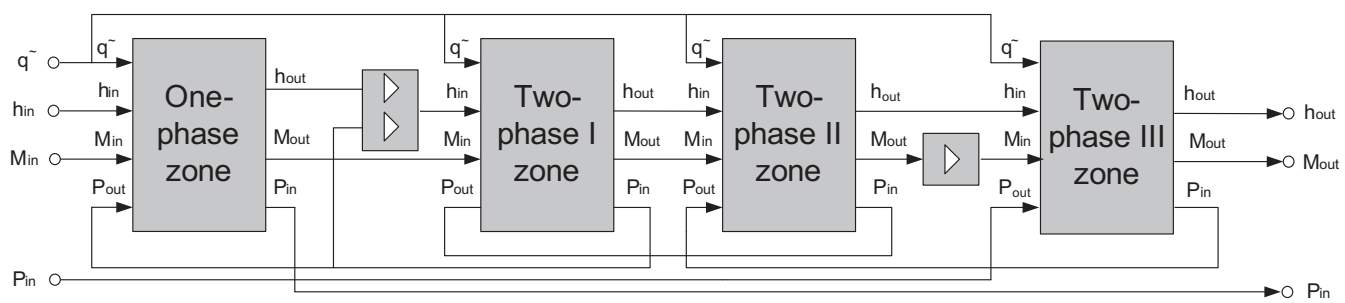

Figure 15. Evaporating tubes model structure

As the reduced models of one-phase zone and the individual parts of two-phase zone approximate correctly the frequency response only for $\omega<20[\mathrm{rad} / \mathrm{s}]$, approximation errors for higher frequencies were not considered as a criterion of reduced model choice. It may therefore turn out that after closing loops of feedbacks for reduced models, the evaporating tubes model will be unstable. The models with adequacy scope 20 [rad/s] may therefore be useful only for dynamic phenomena analysis in individual evaporating tubes parts, without possibility to be applied in creating an evaporating tubes model.

Fig. 16 presents frequency response magnitude and phase of open loops for the first, second, and third feedback loops. Open system gain for high frequencies significantly exceeds the value 1 .
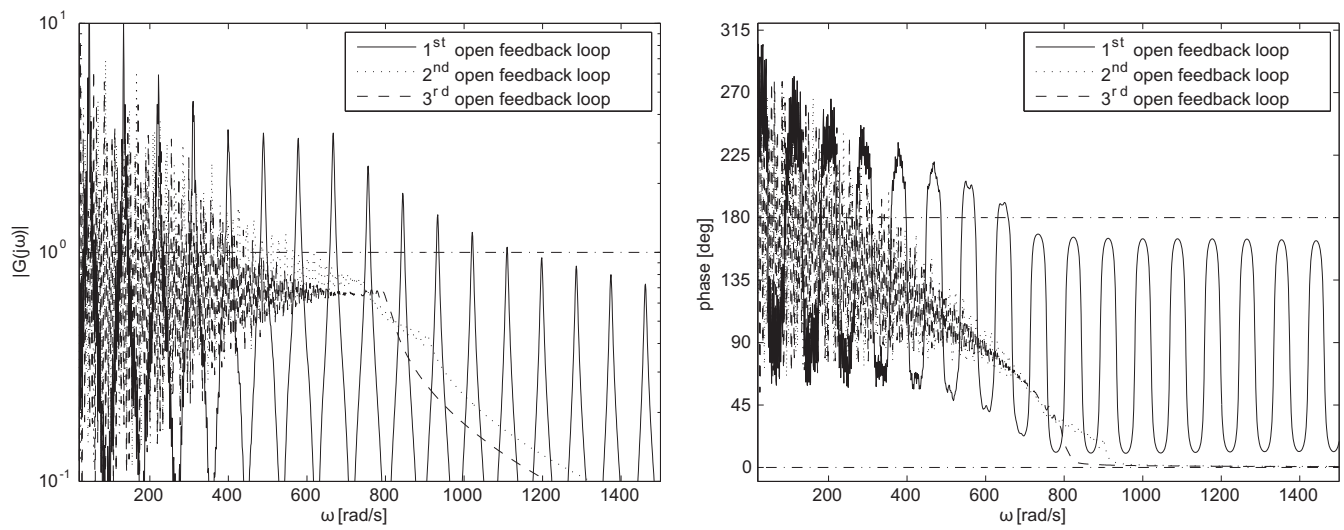

Figure 16. Frequency response magnitude and phase for the first, second and third open feedback loops

A very strong activity of the feedbacks for high frequencies means that in order to guarantee evaporating tubes model stability, obtained by connecting of individual parts 
models, one requires correct approximation of subsystems frequency responses in the scope up to $500[\mathrm{rad} / \mathrm{s}]$, and for the one-phase zone even to 1000 [rad/s] (models orders and approximation errors are presented in Table 1). Such a wide scope of evaporating tubes subsystems models approximation involves their high order and significant complication, at unchanged adequacy scope $0-20[\mathrm{rad} / \mathrm{s}]$. The model of evaporating tubes obtained on the basis of particular zones reduced models connection at approximation scope up to $500-1000[\mathrm{rad} / \mathrm{s}]$, may be then reduced with maintaining adequacy scope of $20[\mathrm{rad} / \mathrm{s}]$.

From the point of view of evaporating tubes model stability, achieved on the basis of reduced models of individual subsystems, it is advantageous to apply reduced models of one-phase zone and individual two-phase zone parts obtained for the adequacy scope $2[\mathrm{rad} / \mathrm{s}]$. Obtained in this way subsystems models are of a low order, which causes, that the frequency responses magnitude is quickly reduced, and after closing feedbacks the evaporating tubes model remains stable. It is unfortunately happening at the cost of limiting the evaporating tubes model adequacy scope to 2 [rad/s].

Each of the reduced subsystems models is characterized with a determined scope of adequacy and approximation error, as a result of connecting reduced subsystems models, therefore a problem of approximation errors effect on the model error at a higher hierarchy level appears. The problem is analyzed on the basis of multiple reduction of the first level subsystems models with the application of evolutionary algorithms and creating an evaporating tubes model on their basis. Table 2 shows median of the mean square of relative evaporating tubes approximation error ratio to the mean error of lower level subsystems, for two scopes of adequacy of subsystems models: 2 [rad/s] and 500 [rad/s]. This results show that the evaporating tubes model error is characterized with an average $1.5 \div 2$ times higher than the lower rank subsystems models errors, at the same adequacy scope. It was however noticed, that it is possible to select such subsystems reduced models which facilitate compensating approximation errors of individual subsystems. As a result, the model of evaporating tubes may be characterized with the same or even smaller approximation error as the subsystems models.

Table 2. Median of the mean square of evaporating tubes model approximation relative error ratio to the subsystems average error

\begin{tabular}{|c|c|c|}
\hline Approximation scope & $2[\mathrm{rad} / \mathrm{s}]$ & $500[\mathrm{rad} / \mathrm{s}]$ \\
\hline$\frac{\Delta_{3 \text { evaporating tubes }}}{\Delta_{3 \text { subsystem }}}$ & 1.64 & 1.45 \\
\hline$\frac{\Delta_{6 \text { evaporating tubes }}}{\Delta_{6 \text { subsystem }}}$ & 1.90 & 1.42 \\
\hline
\end{tabular}

Results of the reduction of the evaporating tubes model, obtained from subsystems reduced models are presented in Table 3.

Fig. 14 presents eigenvalues of the following evaporating tubes models: 
Table 3. Evaporating tubes model reduction results

\begin{tabular}{|c|c|c|}
\hline Adequacy scope & $\omega_{\max }=2[\mathrm{rad} / \mathrm{s}]$ & $\omega_{\max }=20[\mathrm{rad} / \mathrm{s}]$ \\
\hline Reduced model order & 44 & 85 \\
\hline$\Delta_{3}$ Approximation error & $4.5 \%$ & $8.3 \%$ \\
\hline$\Delta_{6}$ Approximation error & $0.6 \%$ & $0.8 \%$ \\
\hline
\end{tabular}

- ETPRM ${ }_{500}$ (Evaporating Tubes Primary Reduced Model with approximation scope $500[\mathrm{rad} / \mathrm{s}])$,

- ETRM $_{20}$ (Evaporating Tubes Reduced Model with adequacy scope of 20 [rad/s]),

- $\mathrm{ETRM}_{2}$ (Evaporating Tubes Reduced Model with adequacy scope of $\left.2[\mathrm{rad} / \mathrm{s}]\right)$.

\subsection{BP-1150 boiler evaporator model}

The evaporator model of BP-1150 boiler includes five input variables: $M_{s t}, q^{\sim}, M_{s a}$, $M_{f w}, h_{f w}$ and two output variables: $P_{s e p}$ and $H_{s e p}$. Structure of the connections of individual evaporator subsystems models is presented in Fig. 5. Heat processes are significant for the evaporator model dynamic properties, therefore the frequency range $\omega \leqslant 2[\mathrm{rad} / \mathrm{s}]$ was taken into account during the evaporator model reduction.

The evaporator model includes two feedback loops which significantly influence evaporator dynamic properties (Fig. 17). The first one describes influence of pressure changes in the separator on the mass flow and enthalpy of the steam-water mixture at the outlet of the evaporating tubes which, in turn cause pressure changes in the separator. This negative feedback is of internal character and results from the phenomena of the steam generation process. The second feedback is related with the changes of inlet enthalpy to the evaporating tubes (caused by the changes of pressure in the separator). It results from the recirculation of the working medium in the evaporator [28] and constitutes a positive feedback. Frequency responses of the open system for the internal and circulation feedback (at closed internal feedback) is presented in Fig. 18.

\subsubsection{Inner feedback}

Influence of the pressure changes in the separator on enthalpy and mass flow in the evaporating tubes outlet appears as a negative feedback. The shape of open system frequency responses indicates differentiating properties, which in practice means that the inner feedback does not work in the steady state. Due to positive pole, resulting from the separator instability, the inner feedback system is unstable. After determining the poles of the open and the closed inner feedback loop it may be stated, that the positive pole becomes reduced by 4 times as a result of inner feedback (see Fig. 18). Negative inner feedback can not make the closed system stable. 


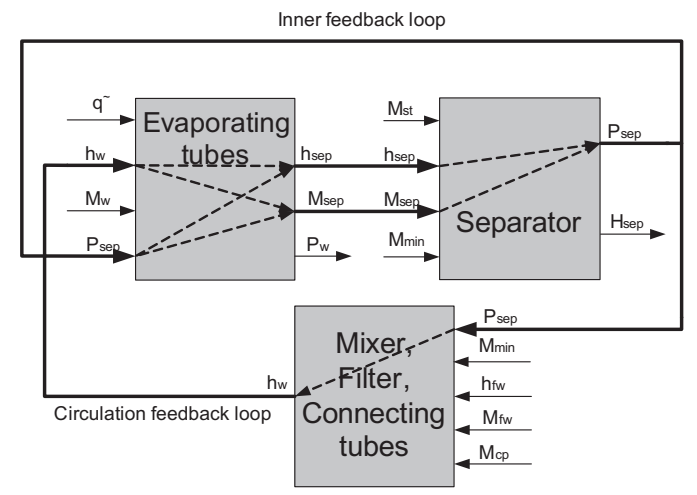

Figure 17. Structure of the inner and circulation feedback in the evaporator
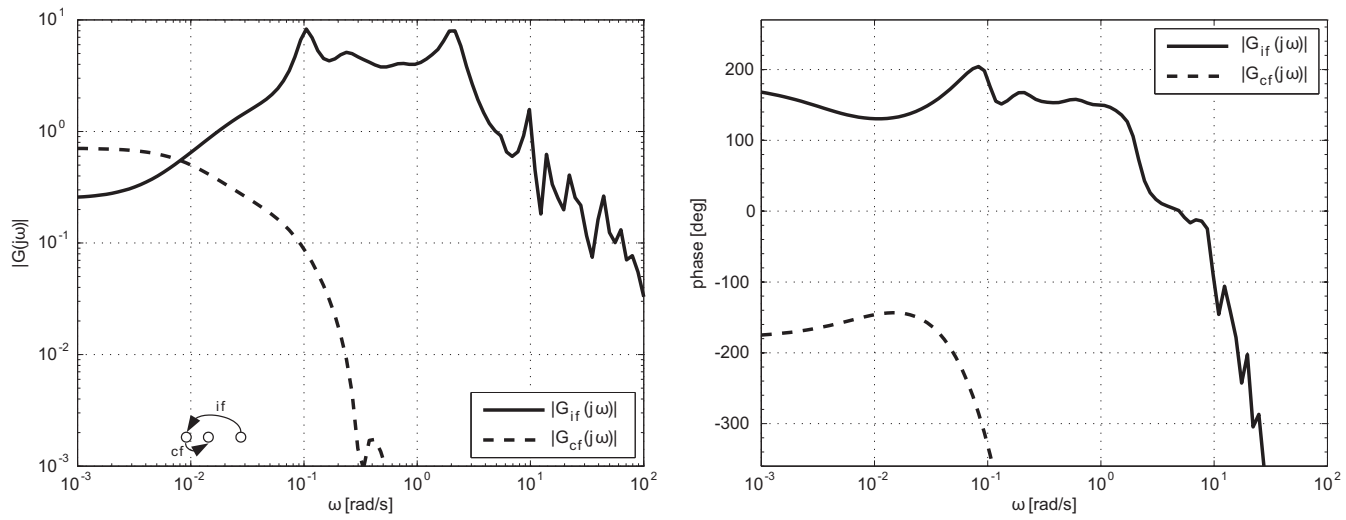

Figure 18. Magnitude and phase frequency responses of the open loop system for internal (if) and circulation (cf) feedback and shifts of the positive pole, as a result of feedbacks

\subsubsection{Circulation feedback}

Circulation feedback loop causes almost double increase of positive pole (Fig. 18). This indicates significant deterioration of dynamic properties of the evaporator. In contrast to the inner feedback, the circulation feedback acts exclusively in the low frequencies range $(\omega<0.1[\mathrm{rad} / \mathrm{s}])$, as faster enthalpy changes are being suppressed in the system of circulation in the evaporator (separator, mixer, filter, connecting pipelines).

\subsubsection{Evaporator model reduction}

Hierarchical model of the evaporator obtained as the result of connecting of reduced subsystems models is characterized with a significant number of state variables. Similarly to the evaporating tubes model, the order of obtained evaporator model can be significantly reduced as a result of further reduction operation. 
In the evaporator model (third level of hierarchy), heat processes are crucial, whereas the dynamic processes related to the working medium transport along the evaporating tubes are practically insignificant. Frequency responses shows that the dynamic processes, taking place in the evaporating tubes, are significantly damped by the separator which is characterized by high heat volume capacity. Separator instability significantly influences the evaporator dynamics, thus the positive eigenvalue in the separator model is also present in the evaporator model (Fig. 19). In the reduced models (e.g. $k=25$ order) the eigenvalues related to the fast mass and pressure transport processes in evaporating tubes are removed, whereas eigenvalues which are responsible for the heat processes remain (Fig. 19).

Fig. 20 presents histogram of the approximation error of the evaporator model related to the average error of the lower level subsystems models. In contrast to the reduced evaporating tubes model, the approximation error of reduced hierarchical evaporator model is smaller than the average error of the subsystems models. This results from small sensitivity of the evaporator model to variations of the subsystems models [25].

The adequacy scope of the obtained evaporator reduced model of the 25-th order is about $2[\mathrm{rad} / \mathrm{s}]$. The model describes processes which are significant for the evaporator dynamic properties and omits fast dynamic processes related to the transfer of pressure and mass flow changes along the long tubes.

Fig. 21 shows the evaporator models set of the BP-1150 boiler and the methods of models creating at individual hierarchy levels. In contrast to the hierarchical evaporator model, in Fig. 22 one level (flat) model of the evaporator is presented, obtained as the result of individual subsystems models connection. The model is of very high order, (over 10000), and its application to the dynamic properties analysis of the evaporator and to the control systems design is practically impossible.

\section{Conclusions}

The control purposes of complex plant need mathematical models which are created by modeling of phenomena occurring in the plant. The complexity of models grows constantly and significantly hinders the usage of the methods and programs for the dynamic properties analysis and the synthesis of control algorithms. Simplicity in attaining complex models follows from application of hierarchical structure and decomposition model into a series of subsystems which are simpler to be modeled. At all higher levels, the hierarchical model contains subsystems included in the lower level with determined topology of connections.

Multi level model structure enables analysis by application of aggregation procedure. One of the principal aggregation resources is reduction of the model at individual levels of hierarchical structure. Such approach allows for creating the reduced hierarchical model including a collection of models at every level of hierarchy, characterized by various adequacy scopes and accuracy of the plants features approximation. 


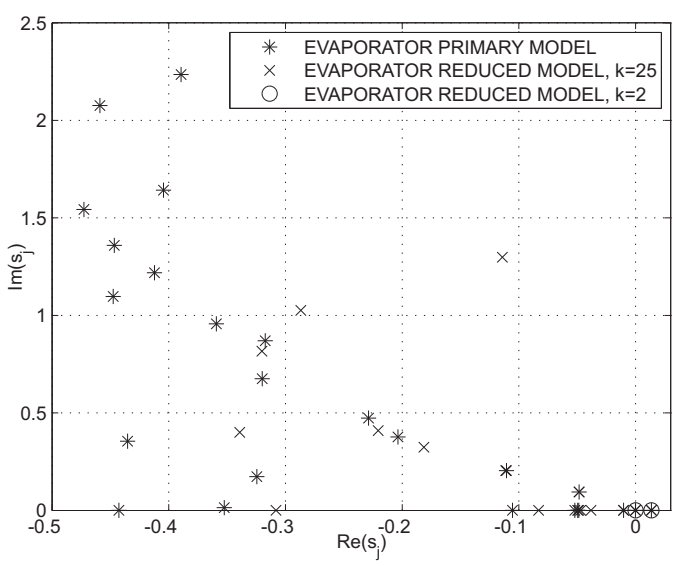

Figure 19. Evaporator model eigenvalues

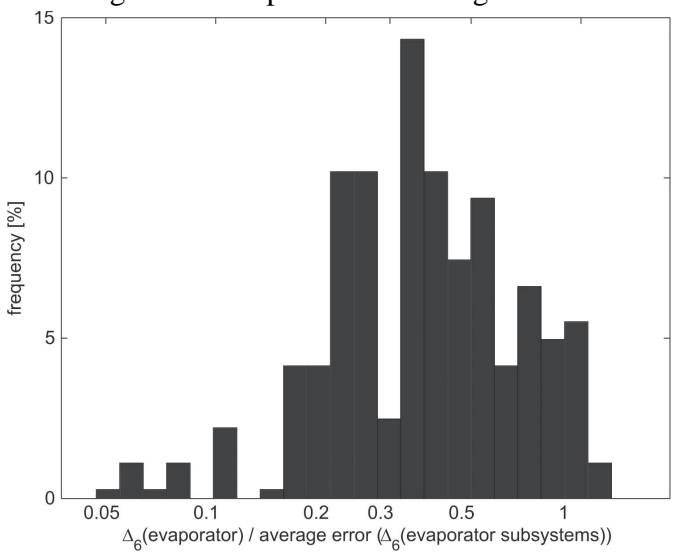

Figure 20. Histogram of the approximation error of the evaporator model related to the average error of the lower level subsystems models

Using this methodology, instead of high order model (flat, one level model), a collection of models is obtained, which are placed at individual hierarchy levels. The models represent dynamic processes typical for each hierarchy level, and omit fast dynamic processes significant at lower levels. The higher hierarchy level, the slower dynamic processes is described by the model and the lower is order of the models.

This paper presents methodology of hierarchical complex plants models creation on the example of evaporator of the BP-1150 boiler. Each of the subsystems at individual levels of model hierarchy are a multi-input and multi-output causal systems. The paper analyzes the evaporator dynamic properties in normal conditions of exploitation on the basis of linearized models.

The evaporator model structure presented in the paper includes four hierarchy levels: 


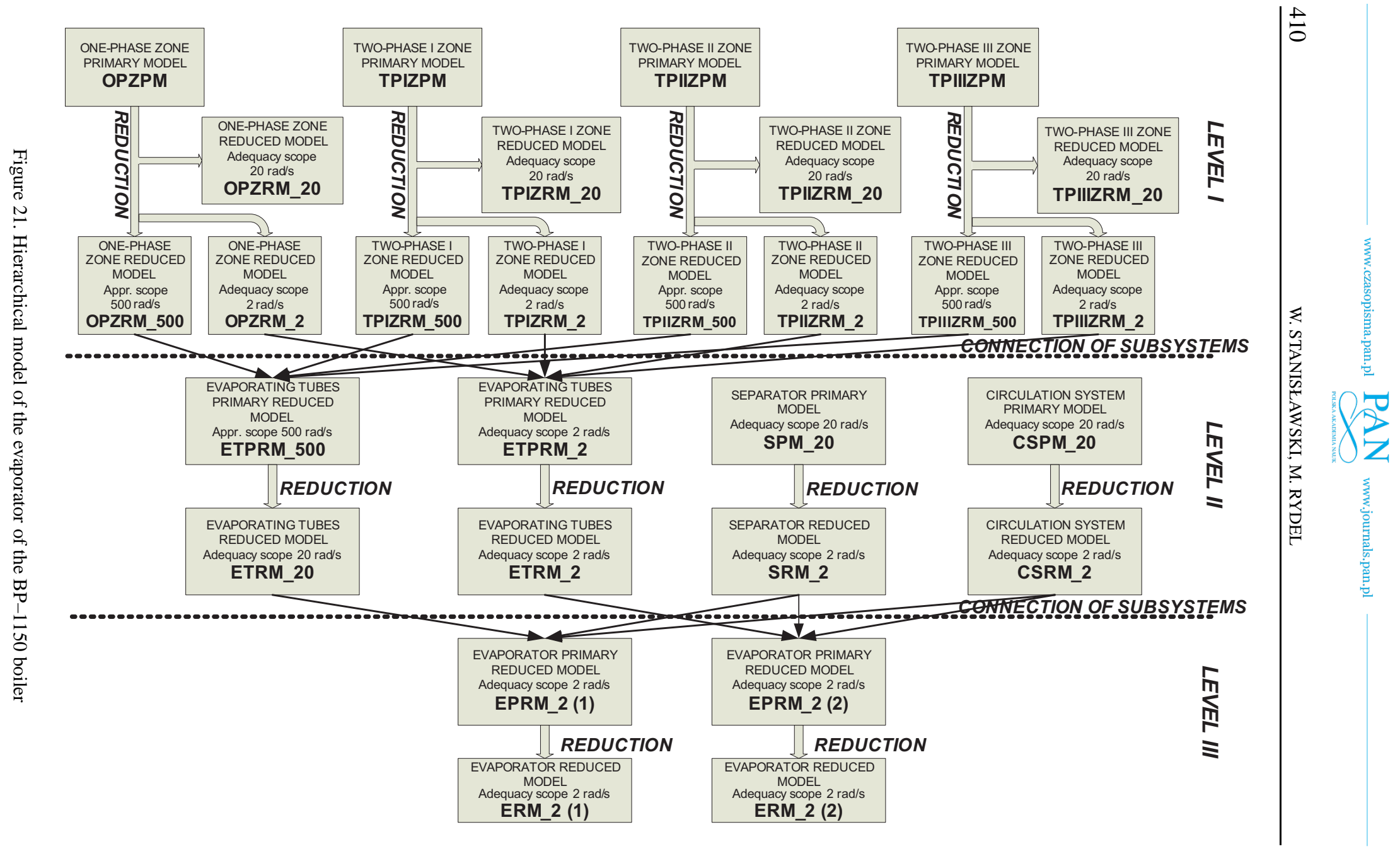




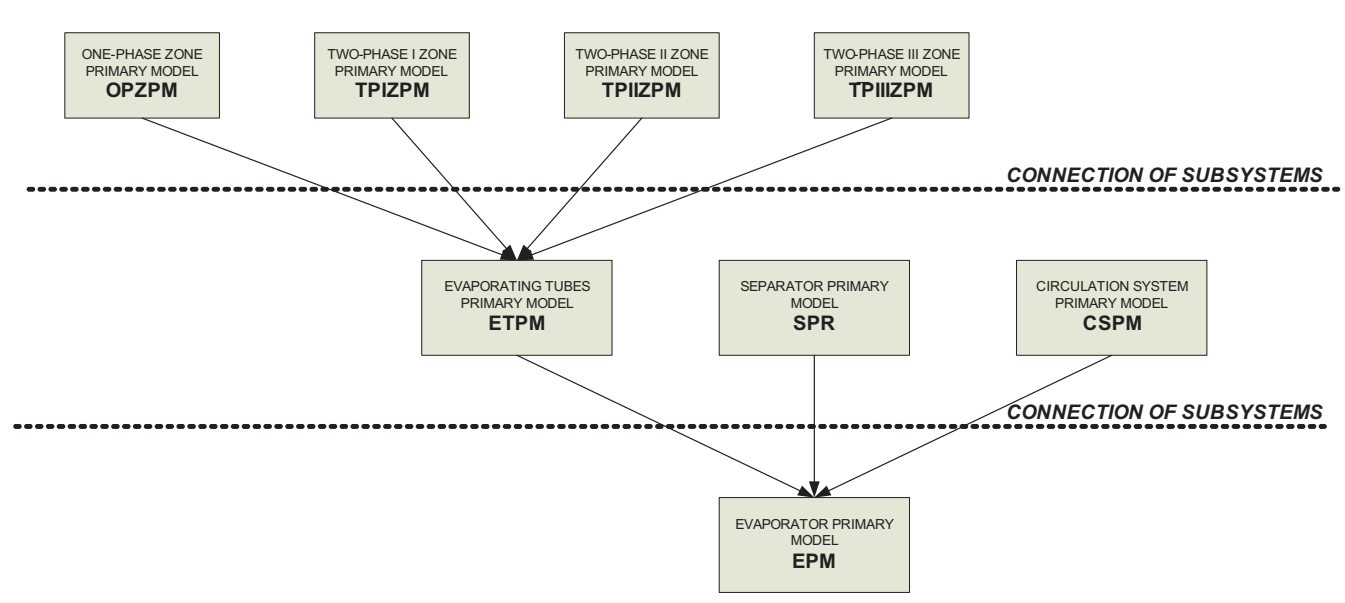

Figure 22. Complex model of the evaporator of BP-1150 boiler obtained on the basis of subsystems models connection

- Level 0 - description with differential and algebraic equations of finite elements which create lumped parameter models.

- Level 1 - individual areas of evaporating tubes defined by working medium flow type - one-phase zone and three parts of two-phase zone: bottom part with large heat load in which most of vapor is being produced, intermediate part with zero heat load and the top part of two-phase zone with little heat load and higher evaporating tubes diameter, which assures higher boiler accumulation capacity.

- Level 2 - evaporator subsystems which include: evaporating tubes, separator, mixer, filter, connecting pipelines and circulation pump.

- Level 3 - once-through evaporator.

Evaporating tubes model of the $\mathrm{BP}-1150$ boiler is described by differential equations system which constitutes the mass, energy, momentum balance as well as the heat conduction along the tube wall and the connecting blade. To assure the adequacy scope of the model with lumped parameters equal $20[\mathrm{rad} / \mathrm{s}]$, it is necessary to apply significant number of finite elements and high model order. Models of individual zones of evaporating tubes are characterized with a very high order: one-phase zone -8250 , two-phase zone I - 2750, two-phase zone II - 4125 and two-phase zone III - 11000 .

As the result of such high model complexity, the basic problem is the choice of proper reduction methods, so that the model complexity is limited, while the dynamic features (significant at a given hierarchy level) are preserved. Out of all presented reduction methods, the best results have been obtained with FW method. 


\section{Appendix A}

\section{Nomenclature}

$\begin{array}{ll}A & \text { cross area }[\mathrm{m}] \\ c & \text { vapor mass fraction [/] } \\ c & \text { specific heat }[\mathrm{kJ} / \mathrm{kgK}] \\ D, d & \text { diameter }[\mathrm{m}] \\ f & \text { pressure drop coefficient }[1 / \mathrm{m}] \\ g & \text { standard gravity }\left[\mathrm{m}^{2} / \mathrm{s}\right] \\ h & \text { specific enthalpy }[\mathrm{kJ} / \mathrm{kg}] \\ h^{\prime}, h^{\prime \prime} & \text { specific enthalpy of boiling water and saturated steam }[\mathrm{kJ} / \mathrm{kg}] \\ H & \text { water level in separator [m] } \\ L & \text { length [m] } \\ M & \text { mass flow rate }\left[\mathrm{kg} /\left(\mathrm{m}^{2} \mathrm{~s}\right)\right] \\ P & \text { pressure }[\mathrm{kPa}] \\ q, q^{\sim} & \text { heat flux [kW/m], relative heat flux }[/] \\ S=w_{2} / w_{1} & \text { slip factor in two-phase flow } / /] \\ t & \text { time [s] } \\ U_{I} & \text { boiling water section displacement velocity }[\mathrm{m} / \mathrm{s}] \\ v & \text { specific volume }\left[\mathrm{m}^{3} / \mathrm{kg}\right] \\ v^{\prime}, v^{\prime \prime} & \text { specific volume of boiling water and saturated steam }\left[\mathrm{m}^{3} / \mathrm{kg}\right] \\ w & \text { fluid velocity }[\mathrm{m} / \mathrm{s}] \\ Z & \text { tube's height }[\mathrm{m}] \\ Z_{I} & \text { boiling water section position }[\mathrm{m}]\end{array}$

\section{Greek symbols}

$\alpha \quad$ heat transfer coefficient $\left[\mathrm{kJ} /\left(\mathrm{m}^{2} \mathrm{sK}\right)\right]$

$\Theta, \vartheta \quad$ temperature $[\mathrm{K}]$

$\rho$ density $\left[\mathrm{kg} / \mathrm{m}^{3}\right]$

$\lambda$ thermal conductivity $[\mathrm{kJ} /(\mathrm{mK})]$ 


\section{Subscripts}

$\begin{array}{ll}\text { bl } & \text { connecting blade } \\ \text { cp } & \text { circulation pump } \\ \text { ct } & \text { connecting tubes } \\ \text { f } & \text { filter } \\ \text { fin } & \text { filter input } \\ \text { fw } & \text { feedwater } \\ \text { in } & \text { inner } \\ \text { m } & \text { mixer } \\ \text { min } & \text { mixer input } \\ \text { sa } & \text { steam attemperators } \\ \text { sep } & \text { separator } \\ \text { st } & \text { steam } \\ \text { tw } & \text { tube wall } \\ \text { w } & \text { water }\end{array}$

\section{References}

[1] A. Antoulas: Approximation of Large-Scale Dynamical System. Society for Industrial and Applied Mathematics, Philadelphia, 2005.

[2] A. Antoulas and D. Sorensen: Approximation of large-scale dynamical system: An overview. Int. J. of Applied Mathematics and Computer Science, 11(5), (2001), 1093-1121.

[3] A. Antoulas, D. Sorensen and S. Gugercin: A modified low-rank smith method for large-scale lyapunov equations. Numerical Algorithms, 32(1), (2003), $27-55$.

[4] P. Benner, E. Quintana-Orti and G. Quintana-Orti: Efficient numerical algorithms for balanced stochastic truncation. Int. J. of Applied Mathematics and Computer Science, 11(5), (2001), 1123-1150.

[5] D. BoLEY: Krylov space methods on state-space control models. Circuits, Systems and Signal Processing, 13(6), (1994), 733-758.

[6] T.Y. CHIU: Model reduction by the low-frequency approximation balancing method for unstable systems. IEEE Trans. on Automatic Control, 41(7), (1996), 995-997. 
[7] D. ENNS: Model reduction with balanced realizations: An error bound and frequency weighted generalization. In 23rd IEEE Conf. on Decision and Control, (1984), 127-132.

[8] P. FELdmann and R.W. FREUnd: Efficient linear circuit analysis by pade approximation via the lanczos process. IEEE Trans. on Computer-Aided Design of Integrated Circuits and Systems, 14(5), (1995), 639-649.

[9] W. Gawronski and J. JuAng: Model reduction in limited time and frequency intervals. Int. J. of Systems Science, 21(2), (1990), 349-376.

[10] K. GLOVER: All optimal hankel-norm approximations of linear multivariable systems and their $1,{ }^{\infty}$ error bounds. Int. J. of Control, 39(6), (1984), 1115-1193.

[11] T. Gudmundsson and A. LAUB: Approximate solution of large sparse lyapunov equations. IEEE Trans. on Automatic Control, 39(5), (1994), 1110-1114.

[12] S. GUGERCIN: An iterative svd-krylov based method for model reduction of largescale dynamical systems. In 44th IEEE Conf. on Decision and Control, (2005), 5905-5910.

[13] S. Gugercin and A. Antoulas: A comparative study of 7 algorithms for model reduction. In 39th IEEE Conf. on Decision and Control, 3 (2000), 2367-2372.

[14] S. Gugercin and A. Antoulas: A survey of model reduction by balanced truncation and some new results. Int. J. of Control, 77(8), (2004), 748-766.

[15] M. ILAK and C. ROWLEY: Reduced-order modeling of channel flow using traveling pod and balanced pod. In 3rd AIAA Flow Control Conference, (2006).

[16] D. ImAEv, M. Rydel and W. StAnisŁAWski: Reduction of flow boiler proper models as control objects. In XVI National Automation Conference, (2008), 198207. (in Polish).

[17] A. Laub, M. Heath, C. Paige and R. Ward: Computation of system balancing transformations and other applications of simultaneous diagonalization algorithms. IEEE Trans. on Automatic Control, 32(2), (1987), 115-122.

[18] J.R. Li and J. WHITE: Reduction of large circuit models via low rank approximate gramians. Int. J. of Applied Mathematics and Computer Science, 11(5), (2001), 1151-1171.

[19] Y. LIU and B. ANDERSON: Singular perturbation approximation of balanced system. In 28th IEEE Conf. on Decision and Control, 2 (1989), 1355-1360.

[20] B. MoORE: Principal component analysis in linear systems: Controllability, observability and model reduction. IEEE Trans. on Automatic Control, 26(1), (1981), 17-32. 
[21] G. Obinata and B. Anderson: Model Reduction for Control System Design. Springer-Verlag, London, 2001.

[22] G. PAPPAS: Hierarchically consistent control systems. IEEE Trans. on Automatic Control, 45(6), (2000), 1144-1160.

[23] T. PenZL: Algorithms for model reduction of large dynamical systems. Linear Algebra and its Applications, 415 (2006), 322-343.

[24] L.T. Pillage and R.A. Rohrer: Asymptotic waveform evaluation for timing analysis. IEEE Trans on Computer-Aided Design of Integrated Circuits and Systems, 9(4), (1990), 352-366.

[25] M. RYDEL: Reduction of hierarchical complex objects models based on steam boiler. $\mathrm{PhD}$ thesis, Opole University of Technology, Opole, 2008. (in Polish).

[26] M. Rydel and W. StanisŁaWski: Problems of a complex plant models reduction. Measurement Automation and Monitoring, 2 (2010), 197-200. (in Polish).

[27] M.G. SAFONOV and R.Y. ChIANG: A schur method for balanced-truncation model reduction. IEEE Trans. on Automatic Control, 34(7), (1989), 729-733.

[28] W. StanisŁawski: Modelling and Computer simulation of Power Boilers flow boilers. Monograph Studies, 124 Opole, 2001, (in Polish).

[29] W. StanisŁaWski and D. ImAeV: Hierarchical approach to the steam boiler modelling and simulation. In 12th European Simulation Multiconference, (1998), 171-175.

[30] W. StanisŁaWski and W. Minkina: Verification of mathematic model of steam boiler of bp-1150 boiler, for control purposes. Measurements Automation Robotics, 3 (1999), 7-10, (in Polish).

[31] W. StAnisŁaWSKI and M. RydeL: Aggregation of hierarchial models of complex control objects based on a power plant. In 11th Int. Conf. on Methods and Models in Automation and Robotics, (2005), 985-990.

[32] W. StAniSŁaWSKi and M. Rydel: Hierarchical models of complex control objects. In XV National Automation Conference, (2005), 149-154, (in Polish).

[33] A. VARGA: Balancing-free square-root algorithm for computing singular perturbation approximations. In 30th IEEE Conf. on Decision and Control, 2 (1991), 1062-1065.

[34] G. Wang, V. Sreeram and W. Q. LiU: A new frequency-weighted balanced truncation method and an error bound. IEEE Trans. on Automatic Control, 44(9), (1999), 1734-1737. 
[35] K. Willcox and J. Peraire: Balanced model reduction via the proper orthogonal decomposition. AIAA Journal, 40(11), (2002), 2323-2330.

[36] P. Wortelboer and O. Bosgra: Generalized frequency weighted balanced reduction. In 31st IEEE Conf. on Decision and Control, 3 (1992), 2848-2849. 